\title{
GEOLOGY AND MINERAL RESOURCES OF THE RUSSIAN MISSION C-1, QUADRANGLE, SOUTHWEST ALASKA
}

By T.K. Bundtzen and G.M. Laird

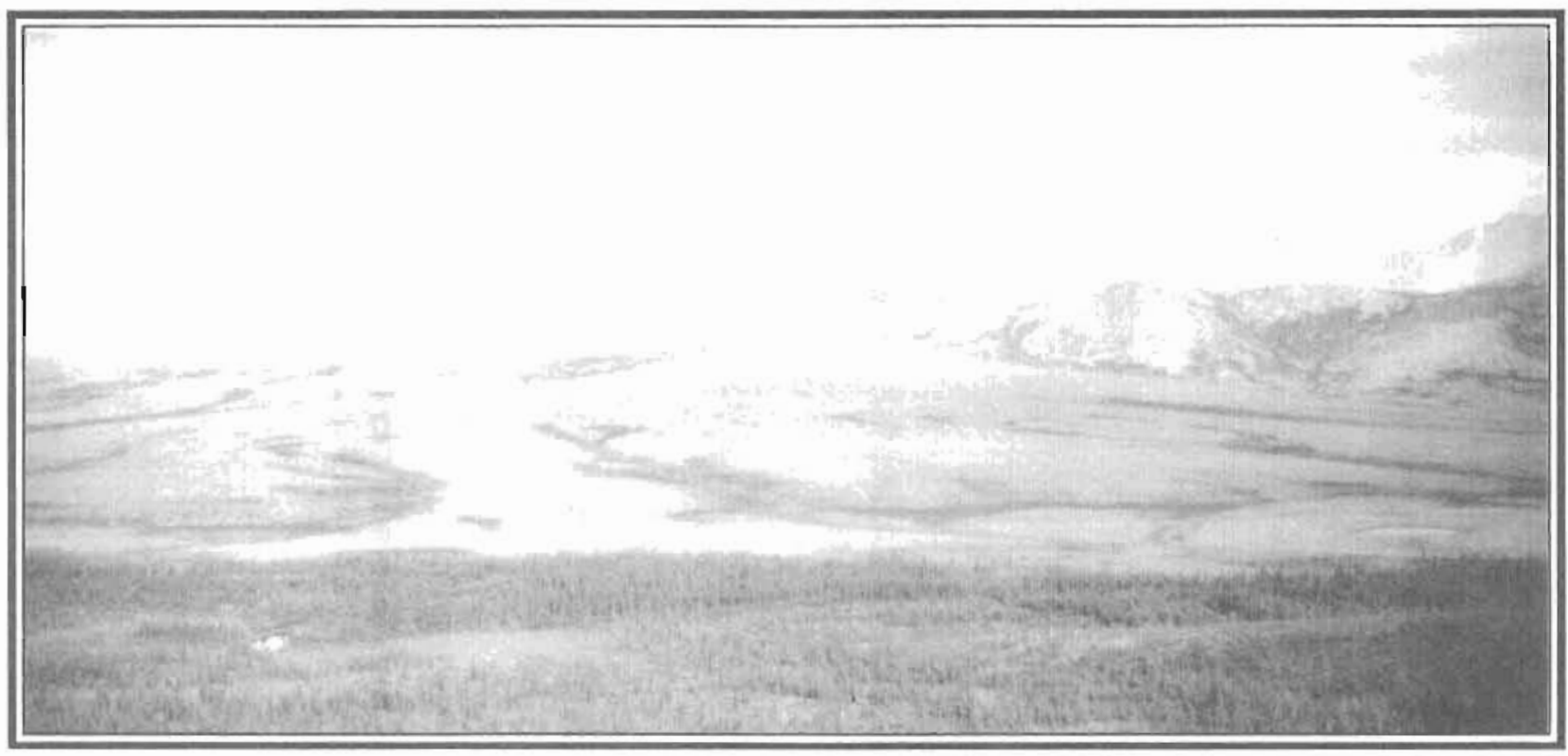

Professional Report 109

Published by

STATE OF ALASKA

DEPARTMENT OF NATURAL RESOURCES

DIVISION OF GEOLOGICAL \& GEOPHYSICAL SURVEYS

Fall

1991 


\section{GEOLOGY AND MINERAL RESOURCES OF THE RUSSIAN MISSION C-1 QUADRANGLE, SOUTHWEST ALASKA}

By T.K. Bundtzen and G.M. Laird

Division of Geological \& Geophysical Surveys

Professional Report 109

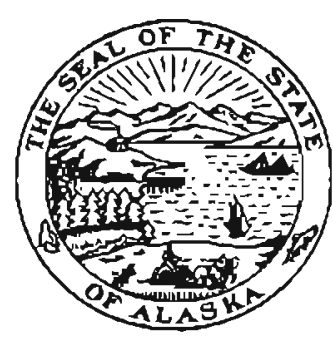

Fairbanks, Alaska

1991 


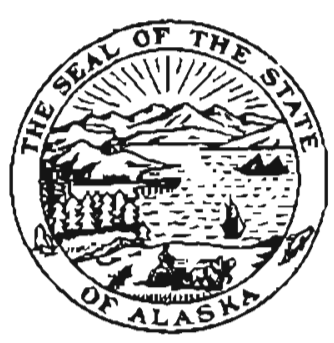

STATE OF ALASKA

Walter J. Hickel, Governor

DEPARTMENT OF NATURAL RESOURCES

Harold C. Heinze, Commissioner

DIVISION OF GEOLOGICAL \& GEOPHYSICAL SURVEYS Thomas E. Smith, Acting Director and State Geologist

DGGS publications may be inspected at the following locations. Address mail orders to the Fairbanks office.

Alaska Division of Geological \& Geophysical Surveys

794 University Avenue, Suite 200

400 Willoughby Avenue, 3rd floor

Fairbanks, Alaska 99709-3645

Juneau, Alaska 99801-1796

U.S. Geological Survey Earth Science Information Center

605 West 4 th A venue, Room G684

Anchorage. Alaska 99501-2299 4230 University Drive, Room 101

Anchorage, Alaska 99508-4664

\begin{abstract}
This pubtication, released by the Division of Geological \& Geaphysical Surveys, was produced at a cost of $\$ 7.50$ per copy which includes the text printed in Fairtanks, Alaska and the oversize ruaps which were primted in Washington, D.C. Publication is required by Alaska Stawte 41, "lo determinc the potential of Alaskan land for production of metals, minerals,... [and] advance knowledge of the geology of Alaska."
\end{abstract}

Cover: View east of outwash fan on East Fork Owhat River, Russian Mountains, Russian Mission C-I Quadrangle, Alaska. 


\section{CONTENTS}

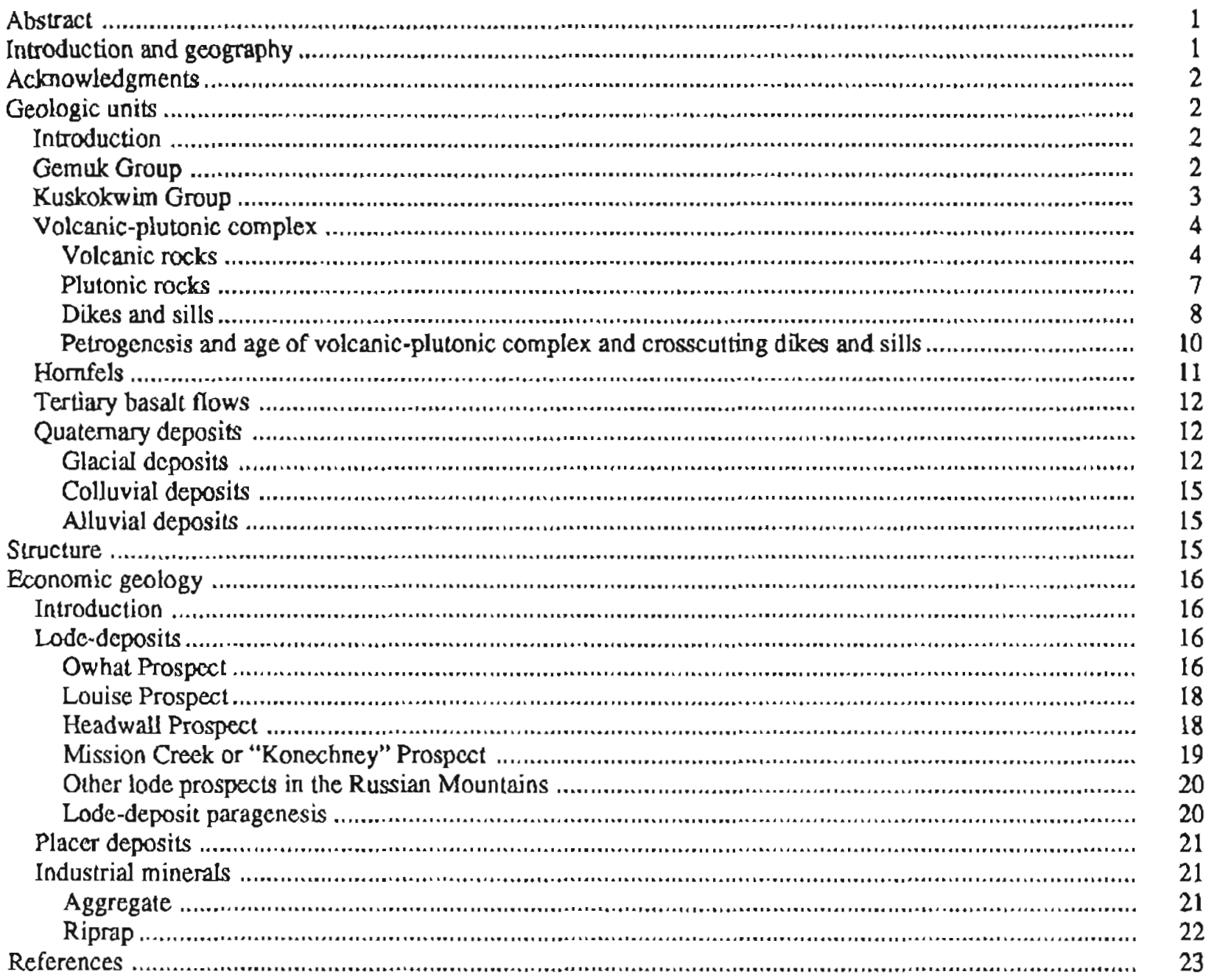

\section{FIGURES}

Figure 1. Lacation map of Russian Mountains and adjacent lowland

2. Photomicrograph of basaliic andesite (85B T175) showing olivine and altered clinopyroxene phenocrysts in groundmass of andesine microlites

3. Plot of Russian Mission C-1 Quadrangle volcanic rocks on $\mathrm{K}_{2} \mathrm{O}-\mathrm{SiO}_{2}$ compositional diagram .......

4. Variation diagram showing chondrite-nomalized rare carth element (REE) concentrations in basaltic-andesite samples from Russian Mountains, Alaska

5. Photomicrograph of zoned plagioclase $\left(\mathrm{A}_{30}\right)$ phenocrysts jacketed at orthoclase in syeno-monzonite (TKsym), eastem Russian Mountains, Alaska

6. Photomicrograph of quanz syenite (TKsy) showing biotite, sericitized albite, and primary(?) aggregates of ferroaxinite

7. Photomicrograph of porfhyritic quartz monzonite (TKmp) showing albite $\left(\mathrm{An}_{12}\right)$ phenocrysts and graphic replacement of K-spar by quartz and plagioclase

8. Photomicrograph of quartz-orthoclase porphyty (TKd) showing gamet, biotite, and sericitized K-spar in undetermined quartz-feldspar groundmass 
9. Normative quartz-K-spar-plagioclase plutonic classification scheme for Late Cretaceous granitic rocks from Russian Mission C-1 Quadrangle, Alaska

10. AFM plot of igneous rocks from Russian Mission C-1 Quadrangle, Alaska

11. Variation diagram showing chondrite-normalized rare-earth element (REE) concentrations in plutonic rocks from Russian Mountains, Alaska

12. Classification of volcanic and plutonic rocks in Russian Mission C-1 Quadrangle using the alkali-lime index of Peacock (1931)

13. Map showing cxtent of Quaternary glacial advances in Russian Mountains, Alaska

14. Photomicrograph of silver-free tetrahedrite along fractures in arsenopyrite from Owhat Prospect

15. Photomicrograph of stephanite (silver-antimony sulfosalt) along fracture in arsenopyrite from Owhat Prospecl

16. Photomicrograph of gold ( 920 fine) and bismuthinite in chalcopyrite from Owhat Prospect

Photomicrograph of arsenopyrite that contains zone of 3.4 percent cobalt and secondary chalcopyrite, from Owhat Prospect

8. Photomicrograph of pekoite in galena, Headwall Prospect

19. Photograph of "Konechney" deposit near Mission Creek Prospect area showing mine dumps and discovery area; view southeast toward Kuskokwim River. Alaska .

20. Plot showing grain-size distribution of outwash of Mission Creek fan, Russian Mission C- 1 Quadrangle, Alaska

\section{TABLES}

Table 1. K-Ar age determinations îor sclected rock samples from Russian Mission C-1 Quadrangle, Alaska

2. Major-oxide determinations and CIPW normative mineralogy for selected igneous rocks from Russian Mission C-1 Quadrangle, Alaska

3. Geochemical determinations of selected samples from prospects and mineral occurtences in the Russian Mission C-1 Quadrangle, Alaska

\section{SHEETS}

[In pocket]

Sheet 1. Geologic map of the Russian Mission C-1 Quadrangle, Alaska

2. Prospect maps of gold-arsenic-copper in the Russian Mission C-1 Quadrangle, Alaska 


\title{
GEOLOGY AND MINERAL RESOURCES OF THE RUSSIAN MISSION C-1 QUADRANGLE, SOUTHWEST ALASKA
}

\author{
By \\ T.K. Bundtzen ${ }^{1}$ and G.M. Laird ${ }^{3}$
}

\section{ABSTRACT}

The Russlan Mission C-1 Quadrangle covers a $522 \mathrm{~km}^{2}\left(20 \mathrm{l} \mathrm{mi}^{2}\right)$ area near the western edge of the Kuskokwim Mountains, an upland dominated by accordant rounded ridges, isolated glacialed massirs, and broad sediment-filled lowlands in southwestem Alaska. The study area is sparsely populated; Chuathbaluk (population 185), is the only communily with year-round residents. The Kuskokwim River flows westward scross the sludy area, marking the divide belween the northem uptands and southem lowlands. The region is dominated by the glacinlly carved Russian Mountains, which rise above lower, rounded hills to a maximum clevation of $1,059 \mathrm{~m}\{3,474 \mathrm{f}\}$.

Bedrock unils range from Penuian or older to late Tertiary in age. The older unit consists of Permian to LowerCretaceous angillite, metabasale, and metasandstone of the Gemuk Group that have undergone lower greenschisl facies metamonthism. In fault contace with the Oemuk Group are Lurbidite-dominated deposils of the Kuskok wim Group, which, based on a sparse fossil assemblage range from latc Early to Late Cretaceous in age. The Kusk okwim Group is about $2,000 \mathrm{~m}(6,500 \mathrm{nt})$ thick in the study area. Intruding and overiying the older layered rocks is an Upper Crelaceous volcanic-plutonic complex of peraluminous, alkali-calcic andesite fows and luff and monzonite to quarz syenite plutonic rocks. In $\mathrm{lum}$, peraluminous felsic dikes and mafic sills also believed to be of Late Cretaceous(?) age intrude the igneous comptex and the older layered rocks. Tertiary basalt caps the Gemuk and Kuskok wim Gronps immediately west of the Owhat River. Quatemary deposits in the Russian Mounlains include four glacisl sequences. Thesc, combined with colian, fluvial, and colluvial deposits, cover about 55 peroent of the map area.

The Owhat fauls, probably a splay of the Iditarod-Nix on transcutrenl fault. trends roughly north-soult along the Owhat River and juxlaposes the Gemuk Group ag ainst the Kuskakwim Group. Most recenl movenient along the fault occurred prior to extrusion of the overlying basalt, which yielded a K-Ar age of $6.19 \mathrm{Ma}$.

Principal mineral depasibs consist of mesolhemal, intrusive-hosted. polymetallic gold-sulfide-tourmaline greisen vcins that intrude a prom:nent high-angle frnclure systens in the ontral Russian Mountains. Fonr prospects contain a minimum inferred reserve of 229.200 metric tons (253.500 shon wons) of ore that grade $4.48 \mathrm{ppm}(0.13 \mathrm{oz} / 10 \mathrm{n}) \mathrm{gold}$, 9.59 percent arsenic, 0.20 percent antimony, 0.01 percent tin, and 0.61 percent copper, and contain anomatous values of silver, lcad, bismulh, lungsten. cobale, zinc, and umsium. Placer gold is present on Mission and Cobalt Creeks, but no mining activily has been recorded. Aggregate sites in the study asca could be developed for local consiruclion projects. A promising riprap quarry site noth of Chuathbaluk slong the flanks of the Russian Mounlains could supply riprap 10 communitics along the Kuskokwim River for erosion-control progrants.

'Division of Genlogical and Geophysical Surveys, 794 Universily Averue, Suite 200, Fajbarkx, Alaska 99705.3645.

\section{INTRODUCTION AND GEOGRAPHY}

During the periods July $23.26,1985$, July 15 and August 11, 1987, and June 12-26, 1988, we conducted 1:63,360-scale geologic mapping projects and related mineral-resource investigations in the Russian Mission C-1 Quadrangle of southwestern Alaska (fig. I). The study area lies within the Kuskokwim Mountains geographic prov. ince, a maturely dissected, generally unglaciated upland that consists of accordant, rounded ridges [averaging eleva-

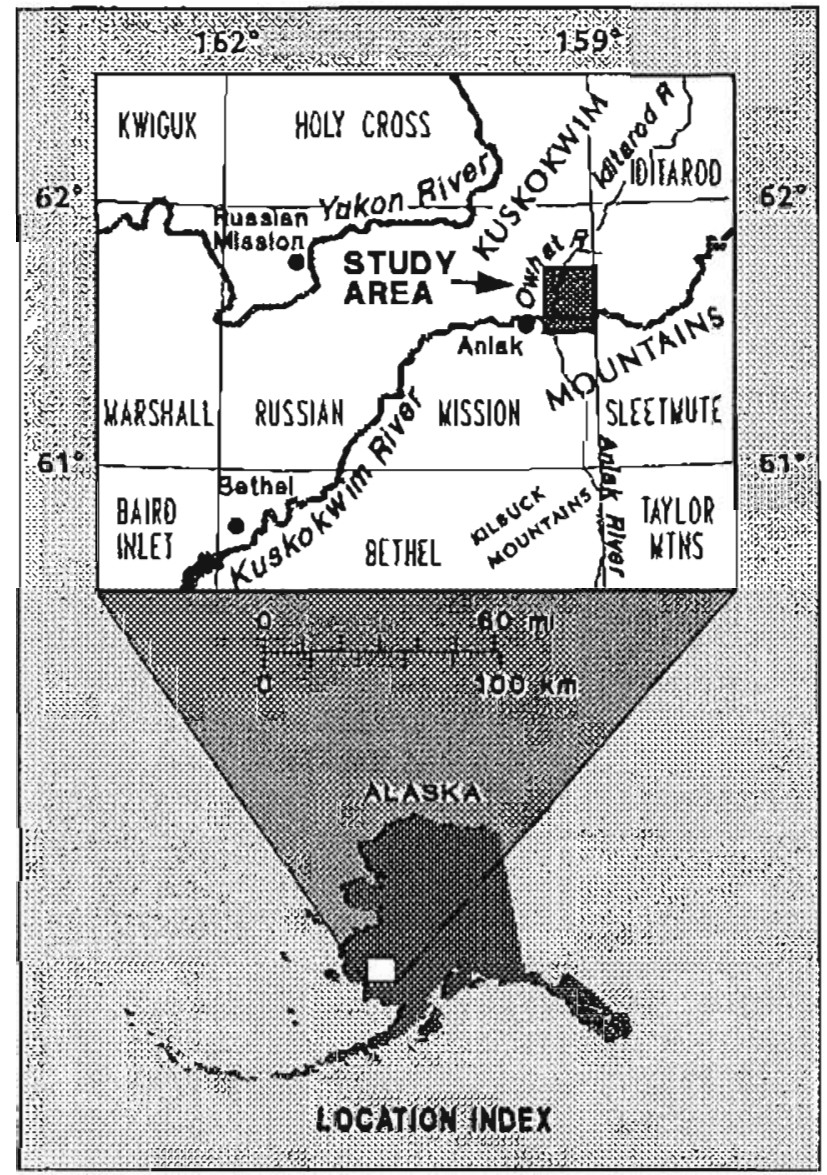

Figure 1. Location map of Russian Mountains and adjacent lowland. 
tion $600 \mathrm{~m}(2,000 \mathrm{ft})]$ interspersed with broad, sedimentfilled lowlands [average elevation $90 \mathrm{~m}$ (300 ft)] (Wahrtaftig, 1965). In the northern half of the quadrangle, rugged peaks of the Russian Mountains massif, composed of granitic, and volcanic rocks, and homfels, cover an area of $140 \mathrm{~km}^{2}\left(50 \mathrm{mi}^{2}\right)$ and rise about $700 \mathrm{~m}(2,300 \mathrm{ft})$ above the surrounding vegetated hills to an elevation of $1.057 \mathrm{~m}$ $(3,468 \mathrm{ft})$. The massif is one of 13 isolated massifs or ridge complexes in the Kuskokwim Mountains that were sufficiently large and high enough to generate alpine glaciers during the Quaternary period (Kline and Bundizen, 1986).

Western and northern slopes of the Russian Mountains are drained by the Owhat River, which flows through low, rolling hills of the $O$ what River upland. Slightly metamorphased sedimentary and volcanic rocks typically underlie the gently rolling hillslopes west of the $O$ what $R$ iver and arc covered with climax white spruce, birch, and sphagnum moss. Bedrock exposures in these lower areas are poor and often confined to river cutbanks. The $O$ what River joins the Kuskokwim River, which is siluatod along the wesicentral boundary [elevation $18 \mathrm{~m}(59 \mathrm{ft})$ ] of the quadrangle. From this point southward, the Kuskokwim River and its flood plain dominate the topography. The Kolmakof River empties into the Kuskokwim River east of the quadrangle and drains most of the eastern slopes of the Russian Mountains. The southern slopes of the Russian Mountains are drained by Mission Creek and other small drainages that also empty into the Kuskokwim River. Quaternary fluvial. colluviah and eolian deposits cover about 55 percent of the study area.

The village of Russian Mission was established along the Kuskokwim River by Moravian missionaries in 1885 (Orth, 1971, p. 821), and rapidly became a major center of the Russian Orthodox Church. Sometime after 1969. the village was renamed "Chuathbaluk" partly because of frequent confusion with the village of Russian Mission located on the lower Yukon River. Today about 185 people live in the community. No thoroughfares have been built in the area aside from a short secondary road that links Chuathbaluk with an airport. Trails for off-road vehicles are abundant and extensively used, particularly during the winter months. The larger village of Aniak (population 540), situated west of the map area about $30 \mathrm{~km}$ (19 mi) downstream from Chuathbaluk, serves as a center of trade, manufacturing, and transportation for communities of the middle Kuskokwim River area.

Lands of the Russian Mission Quadrangle are owned by state ( 31 percent) and federal (35 percent) governments and native regional corporations (34 percent). The subsurface and surface estate of the central and southern Russian Mountains is owned by Calista Native Regional Corporation. Kuskokwim Village Corporation, another Native enterprise, manages the surface holdings of several Kuskokwim River villages, including Chuathbaluk. Outly- ing western and castern outlier ridges of the Russian Mountains and the Owhat upland are owned by the federal govemment and managed by the U.S. Bureau of Land Management under multiple-use principles. The state of Alaska, which also manages its lands for multiple use (Alaska Department of Natural Resouces, 1986), owns most of the study area south of the Kuskokwim River and the beds of all navigable rivers.

\section{ACKNOWLEDGMENTS}

We thark Wyatt G. Gilbert (DGGS) for assisting us in the freld during July 1987, and William W. Patton, Jr., and John M. Murphy (U.S. Geological Survey) for providing logistical support and technical advice during August 1987. We also thank Bruce Hickok and Mike Niemeyer of Calista Native Regional Corporation for permission to examine the geology of Calista lands in the study area. We appreciate the technical reviews by Richard C. Swainbank (Alaska Department of Commerce and Economic Devclopment, Division of Business Development) and Jefrey T. Kline, Mark S. Robinson, and Diana N. Solie (DGGS), and gratefully acknowledge the dedication of senior manuscript typist Roberta A. Mann (DGGS). We thank Ellen E. Harris for the cartography and for drafting most of the figures in this report.

\section{GEOLOGIC UNITS}

\section{INTRODUCTION}

Fifteen bedrock units, ranging from Pemian to late Terliary in age, are exposed in the study area. Samples of all units were studied by standard petrographic techniques in conjunction with microprobe, major-oxide, and multielement and radiometric analyses (tables 1-3). Fourteen glacial, fluvial, eolian, and colluvial units of Quaternary age were identified by field examination and photogeologic techniques. Abbreviated descriptions of all geologic units are provided on sheet 1.

\section{GEMUK GROUP}

The oldest known bedrock unit in the study area consists of poorly exposed argillite, mafic meta-igneous rocks (mainly metabasalt), and tuffaceous metasandstone believed to be part of the Gemuk Group (sheet 1, MzPzvs). Cady and others (1955) originally described the Gemuk Group for exposures near Cinnabar Creck, aboul $60 \mathrm{~km}$ (40 mi) southeast of the study area. Our descriptions are based on a brief examination of rubble crop west of the Owhat River, which Hoare and Coonrad (1959) originally assigned to the Kuskokwim Group on the basis of photogeology. 
Table 1. $K$-Ar age determinations for selected rock samples from Russian Misstion $C-1$ Quadrangle, Alaska [Analyses by Robin Cotrel1, DGGS-UAF Cosperative Geochronology Laboralory, Filiranka, Alaska]

Map no." (fleld no.)

Rock type:

Mineral dated

Sample we (g)

$\mathrm{K} O(\mathrm{Wr} \%)$

${ }^{\infty} \mathrm{Ar}\left(10^{-11} \mathrm{~mol} / \mathrm{g}\right)$

${ }^{4} \mathrm{Ar}(\%)$

$\frac{\mathrm{Ar}^{\circ}}{{ }_{\mathrm{K} \times 10^{3}}}$

Age (Ma)
1 (88BT113)

Vesicular basait

Whole rock

2.170

0.667

0.5944

37.87

0.36000

$6.19 \pm 0.19$

2 (BSBT174)
Biotite-pyrorene
quartz monzonite
Biotite

0.1197

8.96

92.50

80.58

4.1658

$70.3 \pm 2.1$

\begin{tabular}{l}
3 (888T95) \\
Tuffuceous \\
pyroxene mudesite \\
Whale rock \\
\hline
\end{tabular}

0.8317

4.36

48.51

90.28

4.4960

$75.8 \pm 22$
4 (88BT115)

Themally altered

pyroxene andesito

Plagloclase

1.777

0.608

6.225

93.75

4.1322

$69.8 \pm 2.1 \%$

Sheet 1.

Constants used in nge calculations: $\lambda_{\xi}=0.581 \times 10^{-10} \mathrm{yr}^{1}: \lambda_{\beta}=4.962 \times 10^{-10} \mathrm{yr}^{1}$; and ${ }^{10} \mathrm{~K} / \mathrm{K}_{\mathrm{cose}}=1.167 \times 10^{-1} \mathrm{~mol} / \mathrm{mol}^{2}$.

'Minimum age, $10^{\circ} \mathrm{yr}$.

'Radlogenic isotope.

Thin-section analyses $(\mathrm{N}=3)$ of lithic sandstone from the MzPzus unit revealed subangular to angular grains of quartz (35 percent), angular clasts of chert ( 25 percent), rock fragments other than chert (17 percent), pumpellyite (2 percent), while mica (5 percent), albitized plagioclase (11 percent), chloritc and epidote (6 percent), and indeterminated matrix ( 10 percent). The clasts have been deforned and recrystallized by regional metamorphism, which, based on the secondary mineral assemblage epidote + pumpellyite + chlorite + albite probably reached lower grecnschist facies conditions (Turner, 1968). The metasandstone and associated metasiltstone in the MzPzvs unil exhibit graded bedding that may have resulted from turbidity currents. Interbeddod dark-gray, massive, cherty argilliteconsists of about 95 percent quartz and feldspar and 5 percent graphitic material. Locally the cherty argillite is difficult to differentiatc from fine-grained igneous rocks. Together the metasedimentary rocks compose about 80 percent of the Gemuk(?) Group in the study area.

The remaining 20 percent of the MzPzvs unit consists of dark-green metabasalt and metadiorite present as discontinuous lenses or layers that extend along strike for several kilometers and are 5 to $50 \mathrm{~m}$ (16 to $160 \mathrm{ft}$ ) thick. The mafic meta-igneous rocks contain relict phenocrysts of olivine (aitered to antigorite), highly corroded clinopyroxene, leucoxene, and albitized plagioclase in a groundmass of plagioclase, magnetite(?), and clinopyroxene. One major oxide analyses (table 2: no. 2) of metabasalt indicates an alkali-olivine basalt classification similar to those described from oceanic arc settings. Stratigraphic relations with adjacent metascdimentary rocks of the MzPzvs unit are uncertain due to lack of field exposures.

No fossils were recovered from the metasedimentary rocks, and the meta-igneous rocks were too altered for radiometric dating. Hoare and Coonrad (1959) reported Permian and Permian(?) fossil localities from correlative rocks immediately west of the study area. Box (1983. 1985 ) assigned rocks similar to those of the Gemuk(?) Group to the Hagemeister terrane, which he regards as an Early Cretaceous oceanic island arc sutured to North America during Early to Late Cresaccous time.

\section{KUSKOKWIM GROUP}

The two major stratigraphic units underlying the study area consist of poorly exposed, fine-10- coarse-grained calcareous lithic sandstone and interbedded micaceous siltstone (Ksl) and fine- to coarse-grained, noncalcareous, micaceous lithic sandstone, interbedded siltstone, and minor shate (Kus). Both units are correlative with the Kuskokwim Group, which was originally described by Cady and others (1955). The Kuskokwim Group along the Kuskokwim River is fairly well exposed, but outcrops in upland areas are rare and generally collapsed into piles of frost-riven rubble.

In thin section, the calcareous lithic sandstone (KsI) is composed of quartz (20 percent), chert (15 percent), slate ( 15 percent), calcite ( 10 percent), albite (5 percent), felsic igneous ( 3 percent), and white mica ( 2 per- 
cent) clasts in a calcite matrix (30 percent). Coarsegrained lithic sandstone (Kus) west of the Russian Mountains consists of subangular grains of quartz (35 percent), chert (20 percent), volcaniclastic material (15 percent), and white mica ( 3 percent) in a pseudomatrix of deformed rock fragments (25 percent) and is similar to that of the MzPzvs unit but less recrystallized. Prehnite and chlorite replace albite and white mica in the calcareous lithic sandstone (Ksl) and plagioclase in the noncalcareous lithic sandstone (Kus), suggesting zeolite-facies metamorphism.

According to Bundzen and others (1989), river exposures of the calcareous sandstone and siltstone (Kls) in the southeastern comer of the map area consist of thythmic beds 5 to $100 \mathrm{~cm}$ (2 to 39 in.) thick. Flute casts, rip-up clasts, and Bouma Ta-cintervals in sandstone beds of both units (Ksl, Kus) suggest that Kuskokwim sediments were deposited by turbidity currents. The general lack of contiguous exposures in the study arca limited furcher sludy of sedimentary features.

Assuming the lithic sandstone beds (KsI, Kus) exposed along the Kuskokwim River above Chuadhbaluk are laterally continuous, we estimate a thickness of at least $2,000 \mathrm{~m}$ $(6,500 \mathrm{ft})$ for the Kuskokwim Group in thestudy area. However. the Cretaceous soction along the river could be structurally repeated by unrecognized isoclinal folding. Cady and others (1955) estimated a thickness of $12,000 \mathrm{~m}(40,000 \mathrm{n})$ for a section of Kuskokwim Group exposed upriver from the study area between the villages of Sleetmute and Crooked Creek. Bundizen and Gilbert (1983) and Bundzen and others (1988a) estimated stratigraphic thicknesses from 2,200 to $3,500 \mathrm{~m}$ (7,200 to 11,500 ft) for the Kuskokwim Group to the northeast. which is similar to unit thicknesses assessed in the study area The lithic sandstone units (Ksl, Kus) may be part of the thinner western edge of a northeasterly oriented, subsiding Late Cretaceous continental trough that was dislocated by transcurrent faults (Bundizen and Gilbert, 1983).

Inoceramus shclls of Cenomanian age (earliest Late Cretaceous) were reported by Hoare and Coonrad (1959) from seven localities within the Kuskokwim Group 12 to 16 $\mathrm{km}$ (7.2 to $10 \mathrm{mi}$ ) upriver from Chuathbaluk. Subscquent fossil cvidence suggests that the Kuskokwim Group ranges from Cenomanian to Maastrichtian (latest Late Cretaccous) age; the most abundant fossil collections are of Turonian (early Late Cretaceous) age (Bundtzen and Gilber, 1983; Bundtzen and Laird, 1983; Bundizen and ohers. 1988a; Miller and others, 1989).

\section{VOLCANIC-PLUTONIC COMPLEX}

Intruding and overlying the Paleozoic to Mesozoic stratigraphic units are Upper Cretaccous volcanic and plutonic rocks in the Russian Mountains and mafic to felsic dikes and sills throughout the upland areas (sheet 1). For purposes of the following discussion, the igneous rocks are categorized by modal and normative mineralogy according to classification schemes of Peacock (1931), Hiatanen (1963), Irvine and Barager (1971), Streckeisen (1973), and McBimey (1984).

\section{VOLCANIC ROCKS (TKvi, TKva, TKvm, TKvt)}

Basaltic andesite and andesite flows (TKvm), andesite to dacite flows and tuff (TKvt, TKvi), and volcanic agglomerate (TKva) blanket the northern flanks of the Russian Mountains. Poorly exposed andesite to dacite flows (TKvi) also cover a small isolated area south west of the mountains. Volcanic units weather to black-lichen-covered, slabby rubble that sharply contrasts with larger blocks of underlying and llanking plutonic rocks.

Individual basaluic andesite (TKvm) and andesite (TKvi) flows range from 5 to $20 \mathrm{~m}$ (16 to $66 \mathrm{ft}$ ) thick and display distinctive columnar joints, which suggests that the units were deposited in a subaerial environment. In thin section, plagioclase phenocrysts from both TKvm and TKvi flows show both nomal and reverse zoning. The phenocrysts of placioclase range from $\mathrm{An}_{\text {san }}$ in composition whereas the groundmass averages $A n_{\text {was }}$; the normative average plagioclase composition from four flow rocks is $\mathrm{An}_{52}$ (table 2). Mafic components of the flows include minor titanaugite, lesser olivine, and rare biotite (fig. 2). Most basaltic andesite samples exhibit ophitictexture: samples of the andesite and dacite more commonly show trachytic texture.

Tuff-dominated lithologies of the andesite to dacite flows and tuff unit (TKvt) contain abundant, altered shards and feldspar microlites in layers 5 to $30 \mathrm{~cm}(2-12 \mathrm{in}$.) thick separated by sandstone beds composed of andesite clasts ( 50 percent), quartz (25 percent), and chert (25 percent). In outcrop, angular, cobble-sized fragments near the base of an individual bed or layer fine upward to silt-sized particles. The graded nature of the alternating sandstone and tuffa. ceous layers suggests that much of the volcanic component was deposited by air-fall processes.

Agglomerate (TKva) contains rounded clasts of andesite and sandstone to $150 \mathrm{~cm}$ (59 in.) diam that are rested in zeolitized, green-gray lapilli tuff. The presence of sandstone suggests that the volcanic pile may have been deposited on the eroded surface of the Kuskokwim Group. Agglomerates in the Russian Mountains bear some resemblance to lahar and related deposits of the Iditarod Basalt in the DeCourcy Mountain area, about $50 \mathrm{~km}$ (31 mi) northeast of the Russian Mission C-I Quadrangle (McGimsey McGimsey and Miller, 1988).

Despite limited outcrops of the Russian Mountains volcanic pile, we recognize a recurring regular stratigraphic 
Table 2 Major-aurde detemminations and CIPWynomative mineralogy for selected igneous rocks from Russian Mission C1 Quiadrangle, Alaska

\begin{tabular}{|c|c|c|c|c|c|c|c|c|c|c|c|c|c|c|c|c|}
\hline $\begin{array}{l}\text { Map no. } \\
\text { Feld no: } \\
\text { Rock type (unit) }\end{array}$ & $\begin{array}{c}\text { i } \\
\text { sirrios } \\
\text { Quarte } \\
\text { Pophyry } \\
\text { (TKdo) }\end{array}$ & 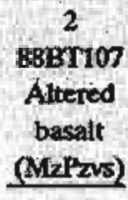 & 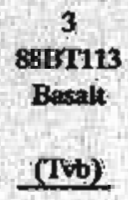 & $\begin{array}{c}4 \\
88 B \text { I95C } \\
\text { Andesite } \\
\text { tuff } \\
\text { (IKVit) }\end{array}$ & 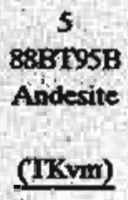 & 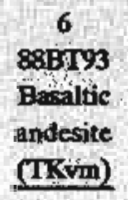 & $\begin{array}{c}7 \\
\text { 8BETrze } \\
\text { Basilicic } \\
\text { andesite } \\
\text { (TKrm) }\end{array}$ & $\begin{array}{c}8 \\
\text { 88BT103B } \\
\text { Quartz } \\
\text { monzonite } \\
\text { (TKmp) }\end{array}$ & $\begin{array}{c}9 \\
\text { 88BTLOBA } \\
\text { Quartz } \\
\text { monzonitite } \\
\text { (TKmp) }\end{array}$ & $\begin{array}{c}10 \\
\text { 88BT67J } \\
\text { Syenite to } \\
\text { monzonite } \\
\text { (TKSym) }\end{array}$ & $\begin{array}{c}11 \\
\text { 88BT67A } \\
\text { Ouartz } \\
\text { serite } \\
\text { (TKSy) } \\
\end{array}$ & $\begin{array}{c}12 \\
88 B 789 \\
\text { Quartz } \\
\text { popphyry } \\
\text { (IXdi) } \\
\end{array}$ & $\begin{array}{c}13 \\
88 \mathrm{GL18} \\
\text { Quart } \\
\text { syentite } \\
\text { (KKY) } \\
\end{array}$ & $\begin{array}{c}14 \\
\text { 88Brs8 } \\
\text { Quartz } \\
\text { senite } \\
\text { (TKSy) } \\
\end{array}$ & $\begin{array}{l}\text { is } \\
88 B 170 \\
\text { Quarte } \\
\text { spenite } \\
\text { (TKSY) } \\
\end{array}$ & $\begin{array}{c}16 \\
\text { 888173 } \\
\text { Ovartz } \\
\text { Ssentite } \\
\text { (TKSy) } \\
\end{array}$ \\
\hline
\end{tabular}

\begin{tabular}{|c|c|c|c|c|c|c|c|c|c|c|c|c|c|c|c|c|}
\hline $\mathrm{SiO}_{2}$ & 67.07 & 45.63 & 5120 & 57.15 & 58.66 & 58.56 & 56.17 & 7.57 & 7269 & 59.66 & 63.95 & 71.16 & 61.89 & 65.22 & 66.37 & 65.90 \\
\hline $\mathrm{Al}_{2} \overline{\mathrm{O}}_{3}$ & 16.38 & 1280 & 14,90 & 20.16 & 15.44 & 17.44 & 16.61 & 14.75 & 14.42 & '16.44 & 16.46 & 16.02 & 17.20 & 16.25 & 15.89 & 16.25 \\
\hline $\mathrm{Fe}_{2} \mathrm{O}_{3}$ & 1.67 & 6.50 & 3.80 & 1.98 & 2.10 & 1.21 & 1.55 & 1.36 & 1.18 & 1.59 & 0.63 & 0.67 & 131 & 0.87 & 130 & 1.07 \\
\hline $\mathrm{FeO}$ & 2.13 & 14,60 & 6.60 & 3.04 & 3.98 & 4.41 & 4.85 & 0.76 & 0.81 & 4.74 & 2.64 & 0.52 & 3.00 & 2.45 & 2.18 & 1.97 \\
\hline Mgo & 0.96 & 241 & 7.20 & 2.26 & 4.97 & 282 & 3.81 & 0.29 & 0.21 & 239 & 1.60 & 0.16 & 1.74 & 1.74 & 1.48 & 0.97 \\
\hline $\mathrm{CaO}$ & 1.42 & 7.85 & 7.77 & 756 & 520 & 4.78 & 5.19 & 0.79 & 0.55 & 4.50 & 2.92 & 2.12 & 3.23 & 280 & 1.92 & 2,00 \\
\hline $\mathrm{Na}_{2} \mathrm{O}$ & 4.85 & 1.77 & 3.47 & 3.33 & 284 & 3.17 & 2.68 & 355 & 3.49 & 2.94 & 3.63 & 4.03 & 392 & 3.43 & 327 & 3.84 \\
\hline $\mathbf{K}_{2} \mathrm{O}$ & 1.64 & 1.03 & 0.70 & 270 & 4.78 & 4.45 & 4.87 & 692 & 6.82 & 4.56 & 6.05 & 3.69 & 5.22 & 6.59 & 6.12 & 5.80 \\
\hline $\mathrm{rio}_{2}$ & 0.57 & 130 & 2.07 & 1.12 & 0.88 & 1.11 & 1.02 & 0.32 & 0.28 & 0.91 & 0.58 & 0.10 & 0.70 & 0.60 & 0.58 & 0.47 \\
\hline$P_{2} O_{5}^{4}$ & 0.26 & 0.79 & 0.28 & 0.60 & 0.42 & 0.52 & 0.50 & 0.08 & 0.07 & 0.41 & 0.26 & 0.07 & 024 & 0.21 & 0.17 & 0.18 \\
\hline Mno & 0.08 & 0.50 & 0.14 & 0.09 & 0.10 & 0.08 & 0,07 & 0.02 & 0.02 & 0.11 & 0.05 & $<0.00$ & $0.0 \%$ & 0.06 & 0.05 & 0.06 \\
\hline Lor" & 267 & 3.00 & $a n$ & 0.54 & $0 . \pi$ & 0.16 & $\mathbf{1 6 8}$ & 0.54 & 0.62 & 130 & 0.55 & 1.73 & 1.66 & 1.02 & 1.49 & 1.35 \\
\hline $\mathrm{CO}_{2}$ & 0.47 & $<0.20$ & $<0.20$ & $<0.20$ & $<0.20$ & $<0.20$ & $<0.20$ & $<0.20$ & $<0.20$ & 1.43 & $<0.20$ & $<0.20$ & $<020$ & $<0.20$ & $<0.20$ & 0.51 \\
\hline Touat & $\overline{100.17}$ & $\overline{98.12}$ & $\overline{98.90}$ & $\overline{100.53}$ & $\overline{100.14}$ & $\overline{87.71}$ & $\overline{99.00}$ & $\overline{100.95}$ & $\overline{101.16}$ & $\overline{100.98}$ & $\overline{99.32}$ & $\overline{10027}$ & $\overline{100.18}$ & $\overline{101.24}$ & $\overline{100.82}$ & $\overline{100.37}$ \\
\hline
\end{tabular}

\begin{tabular}{|c|c|c|c|c|c|c|c|c|c|c|c|c|c|c|c|c|}
\hline Qtrartz & 28.91 & 4.74 & 297 & 8.87 & 6.25 & 7.78 & 4.69 & 22.45 & 24.83 & 10.79 & 10.77 & 29.33 & 8.98 & 11.36 & 17.27 & 15.44 \\
\hline Corundum & 4.81 & 0.00 & 0.00 & 0.00 & 0.00 & 0.00 & 0.00 & 0.17 & 0.46 & 0.00 & 0.00 & 1.74 & 0.00 & 0.00 & 0.81 & 0.46 \\
\hline Othodass & 9.99 & 6.54 & 4.18 & 1596 & 28.42 & 26.68 & 2957 & 40.72 & 40.08 & 27.43 & 36.20 & 22.13 & 31.31 & 38.86 & 36.41 & 34.79 \\
\hline Albitc & 4229 & 15.54 & 29.65 & 28.18 & 24.18 & 2722 & 22,30 & 29.91 & 29.37 & 2532 & 31.10 & 34.60 & 33.67 & 28.96 & 27.85 & 32.98 \\
\hline Anorthite & 5.51 & 26.00 & 23.23 & 32,09 & 1536 & 2051 & 19.43 & 338 & 2.26 & 18.52 & 10.88 & 10.21 & 14.13 & 9.46 & 8.47 & 8.88 \\
\hline Diopside & 0.00 & 8.84 & 11.08 & 1.21 & 6.29 & 0.09 & 3.00 & 0.00 & 0.00 & 1.22 & 1.70 & 0.00 & 0.45 & 2.48 & 0.00 & 0.00 \\
\hline Hypersene & 4.26 & 23.61 & 18.70 & 731 & $13 \pi$ & 1258 & 14.53 & 0.72 & 0.61 & 31.65 & 6.70 & 0.66 & 7.63 & 6.01 & 5.79 & 4.55 \\
\hline Magnetite & 250 & 10.12 & 5,56 & 2.87 & 3.06 & 1.78 & 231 & 1.84 & 1.70 & 235 & 0.92 & 0.99 & 1.93 & 1.26 & 1.90 & 1.58 \\
\hline Imenite & 1.12 & 2.65 & 3.97 & 2.13 & 1.68 & 2.14 & 1.99 & 0.60 & 0.53 & .176 & 1.12 & 0.19 & 1.35 & $\begin{array}{l}1.14 \\
1.14\end{array}$ & $\begin{array}{l}1.111 \\
1.01\end{array}$ & 0.91 \\
\hline Apatite & 0.62 & 1.96 & 0.66 & 1.39 & 0.98 & 122 & 2.19 & 0.18 & 0.16 & 0.97 & 0.61 & 0.16 & 056 & 0.48 & 0.40 & 1.42 \\
\hline Total & $\overline{100.01}$ & 100.00 & $\overline{100 . \infty}$ & $\overline{100001}$ & 99.99 & $1 \overline{100.00}$ & $\overline{100.01}$ & $\overline{99.97}$ & $\overline{100,00}$ & $\overline{100.91}$ & $\overline{100.00}$ & 100.01 & $\overline{100.01}$ & $\overline{100.01}$ & $\overline{100.01}$ & 101.02 \\
\hline Differentiation index & 81.19 & 26.82 & 36.79 & 53.00 & 58.85 & 61.68 & 57.56 & 93.09 & 94.28 & 63.54 & 78.06 & 86.05 & 73.95 & 79.78 & 8153 & 83.21 \\
\hline $\begin{array}{l}\text { Plagioclase } \\
\text { composition (An) }\end{array}$ & $11.52^{\circ}$ & 6259 & 43.94 & 53.24 & 38.84 & 42.98 & 45.47 & 10.16 & 7.14 & 42.24 & 25.93 & 22.78 & 29.56 & 24.62 & 23.32 & 21.21 \\
\hline
\end{tabular}

asheer 1.
Loss on ignition. 
Table 3. Geochemical determinations of selected samples from prospects and mineral occarrences in the Russian Mission C-1 Quadrangle, Alaska and induced nuclear activation leckniques by Bondar-Clegg, Vancowver, British Columbia, Canada. Undertined samples considered anomalous by data inspection

\begin{tabular}{|c|c|c|c|c|c|c|c|c|c|c|c|c|c|c|c|c|c|c|c|c|}
\hline $\begin{array}{l}\text { Ma } \\
\text { no: }\end{array}$ & $\begin{array}{l}\text { Field } \\
\text { no. }\end{array}$ & $\mathrm{Cu}$ & $\begin{array}{l}\text { Po } \\
(\%)\end{array}$ & $\mathrm{Zn}$ & $\begin{array}{c}\mathrm{Au} \\
(\mathrm{ppb})\end{array}$ & $\begin{array}{c}\mathrm{Ag} \\
(\mathrm{ppm})\end{array}$ & As & (\%) ${ }^{\text {Sb }}$ & Mo & Sn & w & $\mathbf{N i}$ & $c_{0}$ & $\begin{array}{l}\mathrm{Cr}^{2} \\
(\mathrm{ppm})\end{array}$ & $\mathrm{u}$ & $\mathrm{Th}$ & La & $\mathrm{ra}$ & cd & Remarks \\
\hline 1 & B8GL31 & .. &.- & 0.01 & ND & ND & $<0.01$ & $<0.01$ & 10 & ND & ND & ND & 6 & 140 & 20 & 5 & 22 & ND & .. & Ferruginous breccia in felsite \\
\hline$\frac{1}{2}$ & 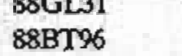 & -. & . & NDD & 8 & $\mathrm{ND}$ & 0.01 & 0.01 & ${ }_{7}^{10}$ & 10 & ND & ND & 3 & 180 & 8.0 & 28 & 3 & 1 & .. & Perruginous syenite \\
\hline 3 & 85BT174 & 0.02 & 0.94 & ND & ND & 45 & 0.46 & 0,30 & -. & ND & ND & .. & .. & 700 & 41.0 & 9 & $\ldots$ & $\therefore$ & 900 & Quartz veinlets in syenite \\
\hline & $\$ 8 B T 24$ & 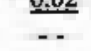 & $\frac{\pi . m}{*}$ & 0.10 & 36 & $\frac{25}{75}$ & $\frac{0.07}{0.27}$ & $\frac{0.02}{0.02}$ & ND & 25 & ND & ND & 4 & $\frac{100}{210}$ & $\frac{50}{7.0}$ & 12 & 32 & ND & $\ldots$ & Shear zooe in andesite \\
\hline 5 & $88 B T Y 3 C$ & $\ldots$ & $\ldots$ & $\frac{0.07}{0.01}$ & 180 & $\frac{5}{7}$ & 0.02 & ND & 6 & 10 & ND & ND & 17 & 310 & 5.0 & 10 & 36 & ND & .. & Columnar-jointed pyroxent andesite \\
\hline 6 & $85 B T 178$ & $<0.01$ & $<0.01$ & $\mathrm{ND}$ & $\frac{100}{N D}$ & ND & $<0.01$ & 0.002 & $\therefore$ & ND & 5 & .- & .. & 80 & 7.0 & 14 & -- & -. & ND & Ferruginous shear zone in andesite \\
\hline 7 & $858 T 180$ & $<0.01$ & $<0.01$ & ND & ND & 2 & $<0.01$ & 0.004 & - & ND & ND & .. & -. & 310 & 5.0 & 11 & .. & $\ldots$ & ND & Perruginous shear zone in hornfelsed andesite \\
\hline 8 & 88BTY1 & $\ldots$ & 80.04 & $<0.01$ & 15 & ND & 0.03 & $<0.01$ & No & 5 & 25 & ND & 8 & 230 & 6.0 & ${ }_{14}^{14}$ & 23 & ND & -. & Pan concentrate, Cobalt Creek \\
\hline 9 & 88 BT92 12 & $\ldots$ & -- & 0.04 & 68000 & ND & $\frac{0.05}{0.04}$ & 0.07 & 16 & 1500 & 130 & 500 & 62 & 930 & 16.0 & 22 & 66 & 16 & .- & Pan concentrate, Cobalt Creek tributary fan \\
\hline${ }_{10}$ & 88BT101 & $\therefore$ & $\therefore$ & $\frac{0.01}{0.01}$ & $\frac{0.001}{N D}$ & $\mathrm{ND}$ & $<0.02$ & $<0.01$ & $\mathrm{ND}$ & $\frac{N \mathrm{~W}}{\mathrm{ND}}$ & $N D$ & $\frac{N D}{N D}$ & $\frac{20}{31}$ & 620 & 4.0 & 6 & 19 & $\frac{10}{N D}$ & .. & Euhedral quart in vuess of basaltic andesite \\
\hline 11 & 886221 &.- &.- & ND & ND & $\mathrm{ND}$ & 0.01 & 0.01 & ND & 300 & $\mathrm{ND}$ & ND & 4 & 310 & 6.0 & 11 & 14 & 1 & .. & Ferruginous shear zone in peraluminous felsite \\
\hline 12 & 88BT68 & $\therefore$ & $\therefore$ & 0.07 & 4,100 & $\mathrm{ND}$ & 10.00 & 0.99 & ND & $\frac{1,200}{1,200}$ & ND & $\mathrm{ND}$ & 40 & 200 & $\mathrm{ND}$ & ND & $\mathrm{ND}$ & 8 & .. & Assemopyrite-challoopyrite-quartt vein in greisen, Louise Proppect \\
\hline 13 & 88 BT67A &.- & $\therefore$ & $\frac{0.07}{0.05}$ & 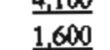 & 120 & $\frac{20.01}{280}$ & $\frac{0.32}{0.21}$ & ND & $\frac{\frac{1, a t u}{N D}}{\mathrm{ND}}$ & ND & ND & 8 & 500 & ND & 3 & 8 . & $\frac{2}{5}$ & -. & 45-in. chip channel in vein, Outbat Prospect \\
\hline & $88 \mathrm{BT} 67 \mathrm{~B}$ & -- & $\therefore$ & $\mathrm{ND}$ & 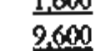 & $\frac{120}{460}$ & $\frac{2.00}{23.00}$ & 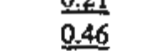 & ND & ND & ND & ND & 79 & 300 & ND & ND & ND & ND & -- & 48-in. chip channet in sulfide greisen vein, Owhat Prospect \\
\hline & $88 B T 67 \mathrm{C}$ & .. & $\therefore$ & ND & $\frac{2000}{9500}$ & $\frac{\mathrm{NO}}{\mathrm{ND}}$ & $\frac{6.00}{26.00}$ & $\frac{0.00}{0.29}$ & $\mathrm{ND}$ & ND & 340 & ND & $\frac{17}{170}$ & 300 & ND & 25 & No & ND & .. & 32-in chip chanel in sollide greisen vein, Owhat Prospect \\
\hline & $88 \mathrm{BT} 67 \mathrm{~T}$ & .. & .. & ND & $\frac{1,100}{1,00}$ & $\mathrm{ND}$ & $\frac{20.04}{4.20}$ & $\frac{0.09}{0.39}$ & $\mathrm{ND}$ & ND & ND & 200 & $\frac{100}{10}$ & 7 & No & ND & ND & $\mathrm{ND}$ & .. & Z-in. chip channel in sulfide-quarty vein, Owhat Prospect \\
\hline & $88 \mathrm{BT} 67 \mathrm{E}$ & $\therefore$ & $\therefore$ & ND & 4,000 & ND & $\frac{4.00}{17.00}$ & $\frac{0.05}{0.25}$ & $\mathrm{ND}$ & ND & $\mathrm{ND}$ & 600 & 34 & 290 & 05 & 3 & ND & 5 & .. & 45-in ctip channel in sulfode veta, Owtal Prospect \\
\hline & $88 B T 67 \%$ & $\therefore$ & $\therefore$ & $<0.01$ & $\frac{3,400}{5,400}$ & ND & $\frac{11.00}{20.00}$ & $\frac{0.25}{0.25}$ & $\mathrm{ND}$ & ND & $\mathrm{ND}$ & $\frac{900}{900}$ & $\mathrm{ND}$ & 300 & 0,5 & 3 & $\mathrm{ND}$ & $\frac{z}{11}$ & $\ddot{--}$ & 28-in. chip channel in massive arsenopyrite vein, Owhat Prospect \\
\hline & $88 \mathrm{BT} 67 \mathrm{G}$ & .. & $\therefore$ & 0,13 & $\frac{3,400}{14,000}$ & ND & $\frac{20.00}{33.00}$ & $\frac{0.020}{0.40}$ & ND & 600 & No & 800 & 170 & 110 & $\mathrm{ND}$ & 3 & ND & $\frac{21}{25}$ & .. & 44 in chip ciannel in massive sulfide gretien vein, Owhat Prospect \\
\hline & 88BT67H & $\because$ & $\therefore$ & $\frac{0.13}{0.25}$ & $\frac{17,000}{19,000}$ & ND & $\frac{300}{14.00}$ & 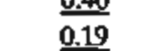 & $\mathrm{ND}$ & 2900 & ND & $\frac{000}{500}$ & 160 & 150 & ND & 2 & $\mathrm{ND}$ & ND & .. & 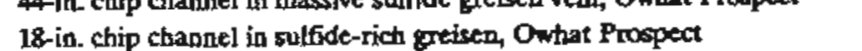 \\
\hline & 88BT67 & $\because$ & $\therefore$ & NDD & $\frac{\frac{17,00}{4,200}}{4}$ & ND & $\frac{1400}{21.00}$ & $\frac{0.00}{0.6}$ & ND & $\frac{N 0}{N D}$ & 320 & $\mathrm{ND}$ & $\frac{1000}{40}$ & 200 & 0.5 & 4 & $<$ & $\mathrm{ND}$ & .. & 38 in. chip chanel in sulfiste-rch greisen, Owhat Prospers \\
\hline & $88 B T 67 \mathrm{~T}$ & $\therefore$ & $\because$ & 0.03 & 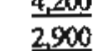 & ND & $\frac{1.00}{1.00}$ & $\frac{0.0}{0.36}$ & ND & & $\frac{200}{N D}$ & 900 & 41 & 250 & 05 & 5 & $<1$ & 7 & .. & 72-in. chip channel in tourmaline greisen, Owhat Prospect \\
\hline & $82 \mathrm{MR} 318 \mathrm{~A}^{\mathrm{b}}$ & 0.21 & 0.04 &.- & $\frac{\frac{1400}{1,240}}{1}$ & 17 & $\frac{1.00}{5.70}$ & $\frac{0.00}{0.06}$ & & $\mathrm{ND}$ & 15 & $\ldots$ & -. & .. & $\ldots$ & .. &.. & $\underline{-}$ & .. & Tourmaline-rich grab sample, Oowat Prospect \\
\hline & $82 M R 318 C^{b}$ & $\frac{0.12}{0.16}$ & $\frac{0.04}{0.06}$ & $\therefore$ & 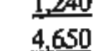 & 85 & $\frac{3 ., 0}{20.80}$ & No & - & ND & 5 & $\cdots$ & -- & .. & .. & -- & $\therefore$ & $\therefore$ & -. & Assenopyrite-bearing quartz-lourmaline vein, Outat Prospect \\
\hline & $82 \mathrm{MR} 318 \mathrm{D}^{\mathrm{b}}$ & $\frac{0.14}{0.14}$ & $\underline{\underline{0.00}}$ & $\therefore$ & 6510 & $\underline{\underline{175}}$ & $\frac{0.00}{33.60}$ & $\mathrm{ND}$ & $\therefore$ & ND & 5 & -- &. & $\therefore$ & -- & - & $\therefore$ & .. & -. & $\begin{array}{l}\text { Massive arsenopyrite with accessory chalcopyrite in toumaline, } \\
\text { Owhat Prosert. }\end{array}$ \\
\hline & 82MR318E & .. & 0.01 & .. & $\mathbf{3 1 0}$ & 6 & 0.24 & 0.01 & .. & $\mathrm{ND}$ & 5 & .. & .. & .. & .. & .. & .. & .. & $\ldots$ & 92-In chip channel in quart-tourmatine vetin, Owhat Prospect \\
\hline & $82 \mathrm{MR} 318 \mathrm{~F}^{b}$ & -- & 0.02 & $\therefore$ & $\frac{300}{1550}$ & 53 & 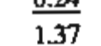 & ND & $\therefore$ & $\mathrm{ND}$ & 15 & .. & .- & .. & .. & .. & .. & .. & & 36-in chip channel in quart-tourmaline vein, Owhat Prospert \\
\hline & $82 \mathrm{MR}^{2} 18 \mathrm{G}^{\mathrm{b}}$ & & $\frac{0.02}{0.01}$ & $\therefore$ & $\frac{\frac{1, N O 0}{N D}}{N D}$ & $\frac{23}{5}$ & $\frac{1.34}{0.47}$ & 0.04 & $\ldots$ & 60 & ND & .. & $\therefore$ & $\ldots$ & .. & .- & .. & .. & & \\
\hline 14 & $88 B T 89 A$ & .. & -. & 0.07 & 470 & $\overrightarrow{N D}$ & $\frac{0.47}{0.41}$ & $\frac{0.04}{0.02}$ & No & $\frac{20}{30}$ & 10 & ND & 3 & 250 & 9,0 & 15 & 16 & 1 & .. & 38-in chip chanolel in greisen, Headwall Prospect \\
\hline & 88BT89B & $\therefore$ & .. & $\frac{0.07}{0.07}$ & $\frac{470}{1,900}$ & $\mathrm{ND}$ & 5.10 & $\frac{0.06}{0.05}$ & ND & $\underline{250}$ & ND & ND & 1 & 110 & 3.0 & 2 & 4 & ND & .. & 27-in. chip channel In arsenopyrite-bearing vein, Headwall Prospect \\
\hline & $88 \mathrm{BT} 89 \mathrm{C}$ & _. & -. & $\frac{0.01}{0.01}$ & $\frac{80}{80}$ & ND & $\frac{\frac{.101}{0.12}}{0.12}$ & $\frac{0.02}{0.02}$ & No & $\frac{52}{25}$ & 25 & ND & 4 & 220 & 17.0 & 25 & 46 & 1 & -. & 85-in. dip chanel in gresisen, Headwall Prospect \\
\hline & 88BT89D & -. & -. & ND & 7900 & ND & $\frac{.12}{1800}$ & $\frac{0.00}{0.20}$ & ND & ND & to & ND & 170 & 310 & 0.5 & 2 & $\mathrm{ND}$ & $\mathrm{ND}$ & & 34-in. chip channel in arsenopyritt-bearing vein, Headwall Prospect \\
\hline & 88BT89F & .. & .. & 0.05 & 9,0000 & ND & $\frac{10000}{2000}$ & $\frac{0.20}{0.70}$ & ND & 2,200 & $\mathrm{ND}$ & 900 & $\frac{10}{7 \pi}$ & 190 & $\mathrm{ND}$ & & ND & 9 & & 7 -in ckjip channel in meisen, Headwall Prospect \\
\hline & 88BT89G & & .. & $\overline{0.01}$ & 210 & $\mathrm{ND}$ & $\frac{0.00}{0.29}$ & $\frac{0.00}{<0.01}$ & No & $\frac{2,200}{15}$ & 20 & $\mathrm{ND}$ & $\frac{5}{30}$ & 310 & 3.0 & 6 & 23 & $\mathrm{ND}^{2}$ & & $\begin{array}{l}65 \text {-in. chip charnel in greisen, Headwall Prospect } \\
6\end{array}$ \\
\hline 15 & $881772 B$ & .. & .. & 0.03 & $\overline{\mathrm{ND}}$ & 7 & $\frac{0.9}{0.61}$ & 0.06 & NO & ND & ND & N & 170 & 410 & 5.0 & 3 & 28 & $N_{D}$ & & $\begin{array}{l}\text { 30-in. chip chanel in tourmatine vein, Mission Creek Prospect } \\
\text { 40-ing }\end{array}$ \\
\hline & $88 B T 72 \mathrm{C}$ & -. & .. & ND & 1,900 & ND & $\frac{0.01}{1.20}$ & $\frac{0.00}{0.70}$ & ND & ND & 55 & 400 & $\mathrm{ND}$ & 360 & 15,0 & 8 & 13 & 5 & & 65-in. chip ctannel in suffide-bearlog vecin, Mission Creek Prospect \\
\hline & $88 B T 770$ & .. & .- & 0.01 & 900 & ND & $\frac{150}{150}$ & 0.12 & $\mathrm{ND}$ & 3 & $\mathrm{ND}$ & $\overline{\mathrm{ND}}$ & 20 & 120 & 10.0 & 11 & 22 & $\overline{1}$ & & Chip sample from greisen, Mission Creek Prospect \\
\hline & $88 B T 72 E$ & .. & .. & 0.03 & 360 & $\mathrm{ND}$ & $\overline{0.77}$ & $\overline{0.7 \pi}$ & ND & ND & ND & ND & 6 & 270 & ND & 28 & 28. & 4 & -. & 24-in. chip channel in greisen, Mission Creck Prospect \\
\hline & 8SBT184A & 3.24 & 0.06 & 0.02 & 8.300 & 26 & 3.00 & 0.21 & $N D$ & 2000 & 20 & .. & .. & 220 & 106 & 3 &.. & -. & 300 & 15-in. chip chamel in greisen, Mission Creck Prospect \\
\hline & 8SBTIBAB & 2.20 & 0.09 & 0.02 & 5580 & 9 & 2.35 & 0.18 & ND & 200 & 10 & .. & -. & 220 & $\underline{\underline{6} .0}$ & 3 & -. & -- & 300 & Yiib-ende ore from mine dump, Mission Creck Prospect \\
\hline & 8SBTI84C & 0.70 & 0.04 & 0.01 & 4,000 & 182 & 1.49 & 0.30 & ND & 100 & ND & .- & -- & 700 & 41.0 & 11 & .. & .- & 900 & 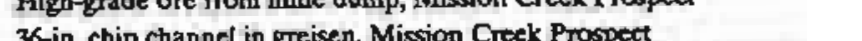 \\
\hline & 85BT184D & $\frac{0.09}{1.99}$ & $\frac{.04}{0.04}$ & 0.02 & 4.907 & $\frac{21}{21}$ & 70.20 & $\underline{0.12}$ & 6 & 34 & 30 & 25 & 5 & $\overline{171}$ &.- & .. & .. & .- & 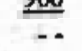 & Grab sample of greisen mineralitation; contains 112 ppm Bi, $12 \mathrm{ppm} \mathrm{Hg}$ \\
\hline & $82 \mathrm{MR}^{2} 15 \mathrm{~A}^{\mathrm{b}}$ & & & & 90 & & 0.66 & 0.012 & $\therefore$ & $\cdots$ & & $\ldots$ & $\ldots$ & 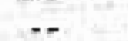 &.- & .. & .. & $\therefore$ & 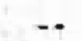 & 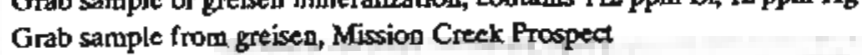 \\
\hline & $82 \mathrm{MR} 315 \mathrm{D}^{\mathrm{b}}$ & .. & .. & .. & 90 & .. & $\frac{0.05}{0.05}$ & 0.03 & .. & .. & .. & .. & .. & .. & -. & -. & .- & -- & .- & Grab sample from greisen, Mission Creek Prospect \\
\hline & $82 \mathrm{MR} 315 \mathrm{~S}^{\mathrm{b}}$ & 0.91 & 0.14 & .. & 18,600 & se & 0.91 & $\underline{0.32}$ & .. & ND & 5 & .. & -- & .. & .. & -- & -. & .. & .. & High-grade- ore with retrograde quartz vein, Mission Creek Prospect \\
\hline & 82MR315F & $\frac{.0}{-.}$ & $\ldots$ & -. & $\frac{\mathrm{ND}}{\mathrm{ND}}$ & $\ldots$ & $\frac{0.02}{0.02}$ & $<0.01$ & -. & -. & .. & .. & -. & -. & -. & .. & -. & .. & .. & Grab sample from greisen, Mission Creek Prospact \\
\hline 16 & $88 B T 76$ & .. & .. & -. & 22 & No & 0.02 & 0.03 & ND & ND & 10 & 900 & 6 & 180 & 14,0 & 21 & 37 & ND & .. & Grab sample from quartz vein with gossan \\
\hline 17 & $88 \mathrm{BT} 75$ & .. & .. & .. & ND & $\mathrm{ND}$ & $\frac{0.02}{0.30}$ & 150 & ND & ND & ND & $\mathrm{ND}$ & ND & 310 & 52.0 & 11 & 54 & ND & .. & Quart vein witt gossan-contains 353 ppal neodymium \\
\hline 18 & $88 G L 36$ & -. & .. & 0.01 & 2200 & ND & 0.01 & $\mathrm{ND}$ & ND & ND & 15 & ND & 30 & 510 & 7.0 & 9 & 81 & ND & -. & Stear zone in gassan horizon of intrusive \\
\hline 29 & $87 G L 37$ & .. & .. & No & $\frac{n}{\mathrm{ND}}$ & ND & $<0.01$ & 0.01 & 7 & 10 & ND & ND & 2 & 100 & 38.0 & 16 & 35 & 1 & .. & Amethyst-bearing vein in homfels \\
\hline 20 & 878TS1 & .. &.- & 0.03 & 250 & $\mathrm{ND}$ & 0.02 & ND & 22 & 60 & ND & ND & 74 & 7500 & $\frac{\frac{0.00}{150}}{150}$ & 11 & 58 & 12 & .. & Grab sample at mine dunp from quartz-bearing vein in bormfels \\
\hline
\end{tabular}




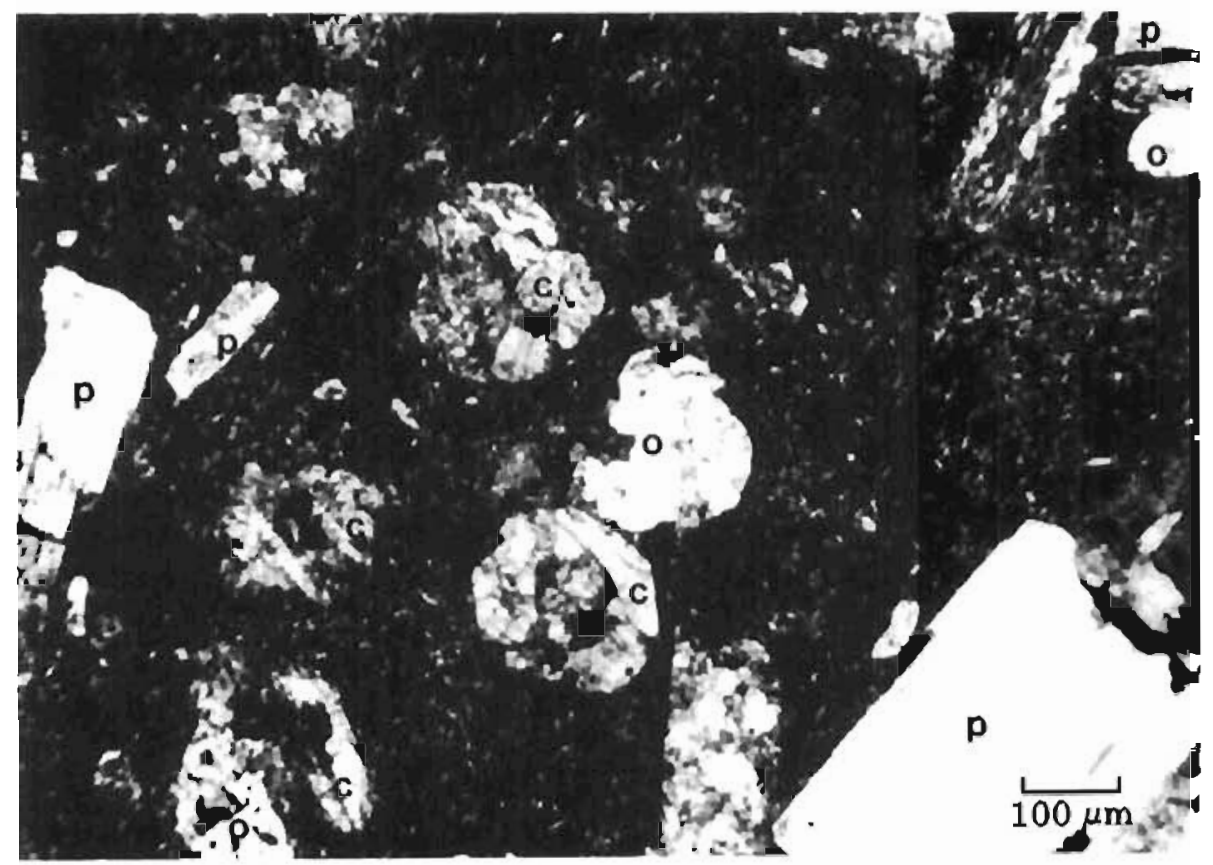

Figure 2. Pholomicrograph of basallic andesile (85BT175) showing olivine (0), plagioclase $\langle p\rangle$, and aliered clinopyroxenephenocrysis (c) in groundmass of andesine microlites, crossed nicols.

pattern (sheet 1). Basal basaltic andesite and andesite flows (TKvm) are overlain by volcanic agglomeralc (TKva), which is capped by andesile and dacile flows and cuff unit (TKvl). This succession-from more mafic hows near the base of the pile to intermediale flows and air-fall-dominated deposits at the lop-is typical of stralovolcanoes worldwide (McBirney, 1984, p. 300-342). The basal basaluic andesite and andesite flows are thickest ( $325 \mathrm{~m} ; 1.065 \mathrm{ft}$ ) in the soulhwestern par of the volcanic pile near VABM 3466 and thinnest to the north and northeast $150 \mathrm{~m}$ (430 $\mathrm{ft}$ ) and $100 \mathrm{~m}$ (330 f1), respectively]. inversely, air-fall and waterlaid tuffs are thickest to the north and northeast $[280 \mathrm{~m}$ (920 fi) and $320 \mathrm{~m}$ (1.050 [1), respectively] and thinnesi $(140 \mathrm{~m} ; 460 \mathrm{ft}$ ) above the thick flow in the southwesters comer of the volcanic buildup. Additionally, clast size in the TKvt unit is coarsesl in the soulhwest ( $f$ to $3 \mathrm{~cm}: 0.510$ $I$ in.) and rines to the north ( $\leq 0.5 \mathrm{~cm}: 0.2 \mathrm{in}$.). We spcculate Wat the flow buildup in the southwest is proximal to the volcanic vent. and that the air-fall turf to the north and norlheast was deposited "downwind" of the volcanic center. The stratigraphic relationship of the poorly exposed andesite to dacite flows (TKvi); (localed south of the Russian Mountains) to the rest of the volcanic pile has not been determined.

The Russian Mountains mafic volcanic rocks (TKvm) lack the iron enrichment typical of tholeiilic suites. Instead, they show ligh alumina and normative plagioclase composilions indicative of calc-alkal ine suites. Moroover, samples from the basalic andesite unit show extreme polassium enrichment, falling within compositional rickds of highpolassium andesite, shoshonite, and banakite (rig. 3). Rareearth-element (REE) trends from three samples of the basaltic andesite (fig. 4) are more or less paraltel, indicate a low degrec of fractionization. and exhibit a modest Eu anomaly. Overall, the petrography, whole-rock chemistry, and REE irends of the Russian Mountains volcanic suite are consistent with those of calc-alkaline to quartz-alkalic igneous belss within ineracontinental back-anc subduction complexes (McBirncy, 1984).

\section{PLUTONIC ROCKS (TKsym, TKsy, TKmp, TKdr, TKdm, TKda)}

The Cretaceous volcanic pile and underlying Kuskokwim Group are intruded by a peraluminous, multiphase pluton (TKsym. TKsy. TKmp) that averages quarz monzonite in composition and by peraluminous quartz porphyry and altered mafic dikes and small intermediate hypabyssal intrusions (TKdf, TKdm, TKda).

The main intrusion. the Russian Mounlairs pluton, crops out in a circular-shaped massif that defines Ihe limits of the Russian Mountains. Intrusive phases are in sharp contact will each other and, along the western, southem, and southeastem margins of the pluton, in sharp high-angle contact with contact-metamorphosed, sedimentary rocks. The Cretaccous volcanic units, often thermally altered, overlie the larger intrusive mass at a low angle; in the northern Russian Mountains, the pluton can be observed through several small windows in the volcanic stratigraphy. Together these contact relations suggest that the entire mounlain range is underlain by intrusive rock (sheet 1).

A syenice 10 monzonite phase (TKsym) generally forms the outermost rim of the Russian Mountains pluton. Because xenoliths of syenite to monzonite are found in 


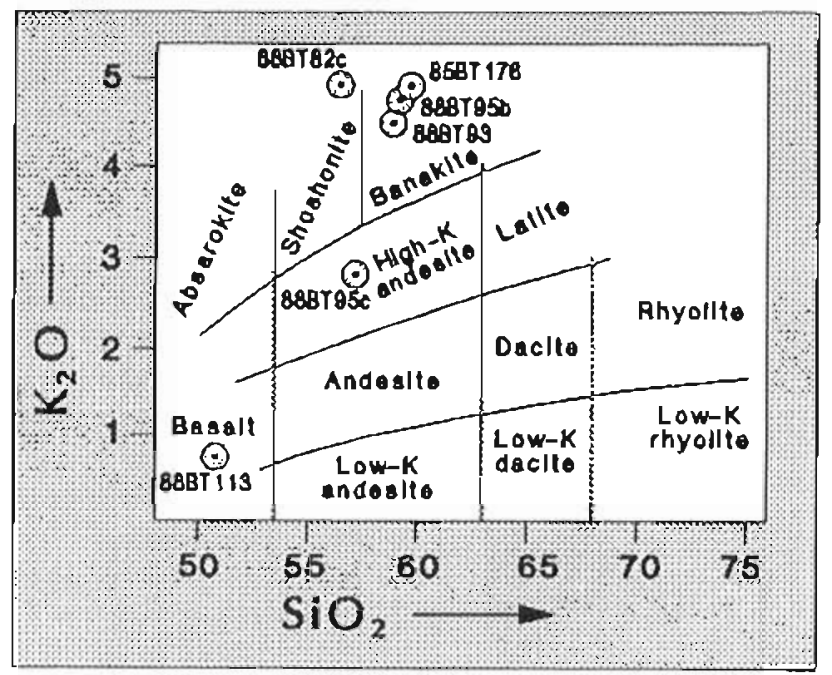

Figure 3.Plol of Russian Mission C-I Quadrangle volcanic rocks on $\mathrm{K}_{2} \mathrm{O}-\mathrm{SiO}_{2}$ composirional diagram (after McBirney, 1984).

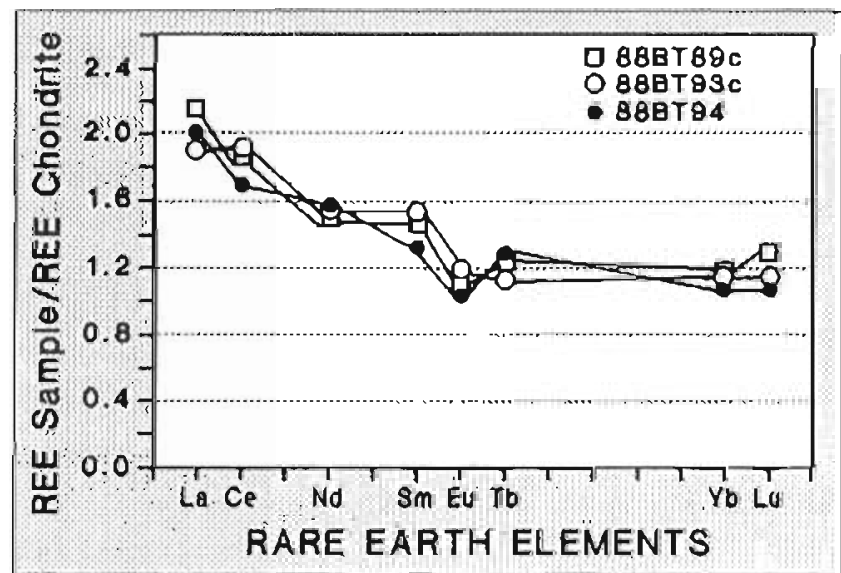

Figure 4. Chondrite-normalizedrarccarthelemen/(REE) concentrations in basalic-andesite samples from Russion Mountains, Alaska. Analyses by Nuclear Activation Services, Onlario, Canada.

quarlz syenite (TKsy) and porphyrilic quarz monzonite (TKmp), the syenite to monzonite is presumed to be the oldest intrusive phase. It generally exhibits a medium- to coarse-grained, hypidiomorphic-granular tex lure and contains zoned plagioclase phenocrysts rimmed by onhoclase (fig. 5) and glomeroporphyritic clusters of altered diopside to $1 \mathrm{~cm}(0.4 \mathrm{in})$ diam in a groundmass of andesine $\left(A n_{33}\right)$, quartz, biolite, and zircon. The immalure lextures suggesl that the syenite to monzonite bodics were emplaced at shallow depths.

Quartz syenite (TKsy), is the most common intrusive phase in the Russian Mountains and comprises aboul 80 pcr- cent of the plutonic mass. Typically, this phase is a light green-gray, medium grained, equigranular to hypidiomorphic rock and consists of orthoclase. oligoclase. quart, and biotile with minor or trace amounts of ( $\geq 1$ percent) diopside. axinite, zircon, and hernatite. In thin section, much of the axinitc appears as discinct grains or platy aggregatesto $5 \mathrm{~mm}$ in diam. The mineral was formed during primary crystallization of the igneous rocks rather than by replacement (fig. 6). This is corraborated by Hietanen and Erd (1978). who previously reporled the presence of primary ferroaxinite in plutonic rocks from the study area and from the McGrath Quadrangle to the northeast. Distinct northwesttrending. linear zones of ankerite-sericite alteration in the valleys of Cobalt and Mission Crecks are probably associated with hydrothermal mineralization along joints and fractures in the quartz syenile.

Porphyritic quartz monzonite (TKmp) crops out as a structurally high intrusive phase in the northwestern Russian Mountains. The unit consists mainly of fune-grained to porphyro-aphanitic, diopsidc-bearing biotite quarz monzonite. Biotile is present as individual grains and as inclusions in altered diopside; subhedral feldspar grains to $2 \mathrm{~cm}$ (0.8 in.) long exhibit rapakivi and graphic textures (Iig. 7). The unit has undergone pervasive hydrothermal alterntion that includes chloritization and epidotization of biotite and diopside, sericitization of feldspar, and silicificalion of the groundmass. Axinite grains and clots $101 \mathrm{~cm}$ (0.4 in.) diam are also present but are tess abundant than in the quartz syenitc (TKsy) unit. Furlher, much of the axinite in the porphyritic quartx monzonite replaces feldspar and biotite.

Although the three intrusive phases (TKsym, TKsy, TKmp) form sharp contacls wilh each other, they arc probably end products of the same differentiation process. The average differcntion indexes for the three phases (TK.sym $=63.5 ;$ TKsy $=79.2 ;$ TKmp = 93.7) suggest that a parent magma of syenite to monzonite composition progressively evolved to quartz syenitc. This is similar to the differenlation sequence described for the Chicken Mountain and Black Creek plutons near Flat, about $72 \mathrm{~km}(45 \mathrm{mi})$ northeast of the Russian Mountains (Bull. 1988: Bundwen and others, 1988b).

\section{DIKES AND SILLS (TKdm, TKda, TKd)}

Dikes and small sills (TKdm. TKda TKdf) intrude along faults and joints in the Russian Mountains pluton and in the older layered units. The most abundant dikes are I to $3 \mathrm{~m}$ (3 109 li) wide, up to $1 \mathrm{~km}$ (0.6 mi) long, and composed of medium-green, chloritized, porphyro-aphanitic, homblende andesite porphyry (TKda) and lighter green quartzorthoclase porphyry (TKdn) (rig. 8). Ubiquitous hydrothermal alieration of homblende in the andesite porphyry 

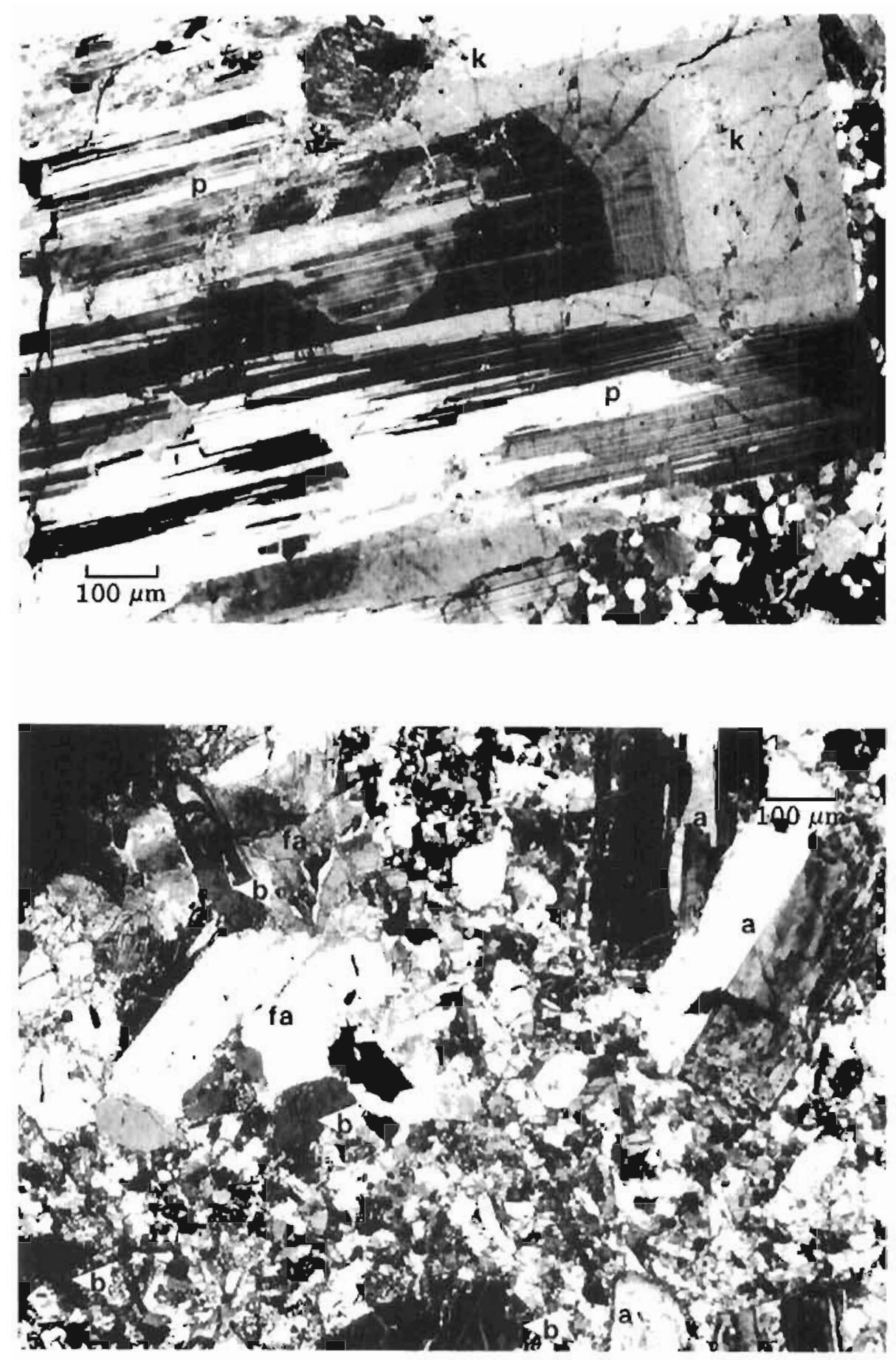

Figure 5. Pholomicrograph of zoned plagioclase $\left(A n_{30}\right)$ phenocrysis $(p)$ jackered byorthoclase ( $k$ ) in syenomonzonite (TKsym). sample 85BT186. eastern Russian Mounlains. Alaska. crossed nicols. prevents K-Ar radiomelric dating; secondarybiotilc clearly posidales homblende and minor pyroxerle bul is ilself altered. Sccondary (?) axinite is also a common constiluent of the andesite porphyry dikes (TKda) that appear 10 be associated with sulfide mineralization (sec "Economic Geology" section).

Quartz-orthoclase porphyry sills $100 \mathrm{~m}$ (328 fl) wide and $3 \mathrm{~km}(2 \mathrm{mi})$ long incrude melasedimencary and mafic meta-igneous rocks (Mzr.Pzus) parallel to the northeasterly structural grain west of the Owhat River. Becausc the felsite silts are compositionally the samc as those intruding the Russian Mountains stock, they are believed to be equivalent.

Minor olivine-clinopyroxene mafic dikes (TKdm) previously described by Bundizen and olhers (1989) crop oul along the Kuskokwim River about $4 \mathrm{~km}$ (2.5 mi) upstream from Chuathbaluk. One prominent system of mafic dikes cuts dark-gray Kuskokwim Group calcareous 
Figure 7. Pholomicrograph of porphyritic quartz monzonire (TKmp), sample 89BTl03. showing albire $\left(A n_{12}\right)$ phenocrysis (a), and ubiquilous graphic replacemeni of $K$-spar by quariz (q) and plagioclase $(p)$, crossed nicols.

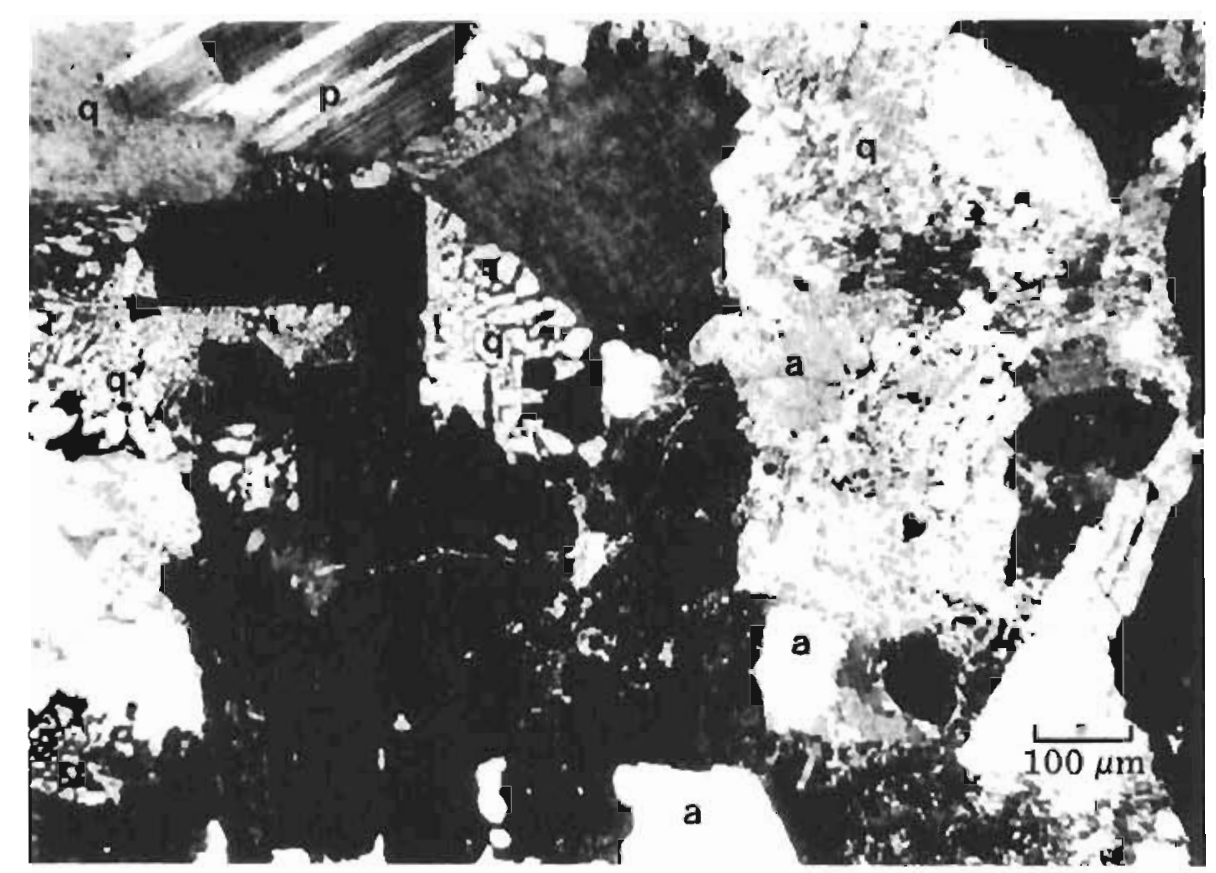

Figure 8. Pholomicrograph of quariz-or/hoclase porphyry (TKuf), sample 88BT109. showing garne' (g), biontite (b), and sericitized $K$-spar (k) in undetermined quarizfeldspar, groundmass. crossed nicols.

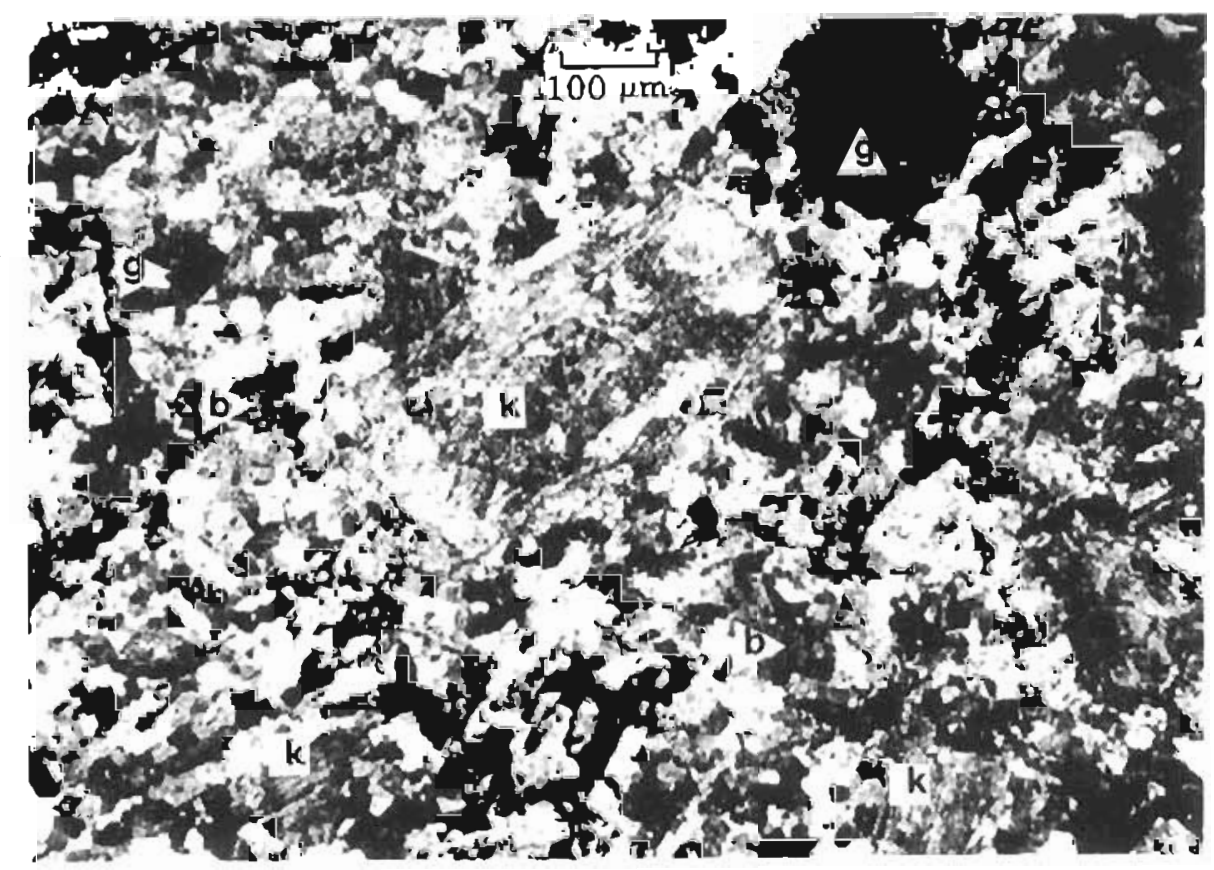

sandstone and siltstone (Ksl). Of these, a 15 - 10 20-m-thick (49 to $66 \mathrm{ft}$ ) dike that trends N. $30^{\circ}$ W. generated enough heat during emplacement to produce a hornfels aureolc $15 \mathrm{~m}$ (49ft) wide, which is unusual for such a small intrusive body. The mafic dike is composed of up to 25 percent cumulus-like grains of olivine and intermeshed clinopyroxene. Unfortunately, no major-oxide determinations are available for the intrusion.
PETROGENESIS AND AGE OF VOLCANIC-PLUTONIC COMPLEX AND CROSSCUTTING DIKES AND SILLS

The Russian Mountains complex is a circular-shaped field of basaltic andesite to dacite tuff $\left(\mathrm{SiO}_{2}=56.17\right.$ to 59.66 percent) that flanks and overlies a monzonite to 
quarte syenite pluton $\left(\mathrm{SiO}_{2}=61.89\right.$ to 72.69 percent). The volcanics are older but probably comagmalic with the pluton. Normative mineral plots show the expected compositional ranges of plutonic rocks (fig. 9), and the expected geochemical evolution of volcanic and plutonic rocks are indicated on an AFM plot (rig. 10). One whole-rock analysis of pyroxene andesite (Lable 1, no. 3) yielded a KAr age of $75.8 \mathrm{Ma}$. The $76 \mathrm{Ma}$ age agrees closely with the $751077 \mathrm{Ma}$ age range obtained from basal parts of the Iditarod Volcanics in the Iditarod Quadrangle $35 \mathrm{~km}$ $(21.2 \mathrm{mi})$ north of the Russian Mountains (Miller and Bunduen, 1988). The volcanics are also similar in age to the 74 Ma maximum age obtained from the Holokuk basalt north of the Kiokluk Mountains about $80 \mathrm{~km}$ (50 mi) southeast of the Russian Mountains (Reifenstuhl and others. 1984; Robinson and others, 1984).

The quarly syenite (TKsy) phase of the Russian Mountains pluton yielded a radiometric age of $70.31 \mathrm{Ma}$ (Lable 1, no. 2). Overall contaci relationships reinforce the contention that the volcanic rocks have been contact metamorphosed and partially assimilated by underlying plutons. Rare-earlt-clement distribution (rigs. 4. I1) atso supports a co-magmatic origin for the plutons and lavas. The alkali-lime index of Peacock (1931) indicates an alkalicalcic classification (fig. 12) similar to other volcanicplutonic complexes studied in the Iditarod and McGrath Quadrangles north of the Russian Mountains (Bundzen and Laird, 1982, 1983; Bundtzen and others, 1988a,b; Bundtzen and Swanson, 1984). Overall, the Russian Mountains volcanic-plutonic complex shows potassium enrichments similar to many shoshonitic suites (McBirney, 1984). The pattem of mineralogy and geochemistry distinguishes the Russian Mountains igneous complex from typical orogenic calc-alkaline magmas. Reifenstuhl and others (1984), Robinson and others (1984), and Decker and others (1984) describe similar volcanic-plutonic rocks, radiometric ages, and geochemistry in the KiokJuk and Chuilnuk Mountains southeast of the sludy area.

\section{HORNFELS}

A pronounced thermal aureole forms a sharp, highangle boundary wilh plutonic rocks along the western, southem, and southeastem limits of the Russian Mountains

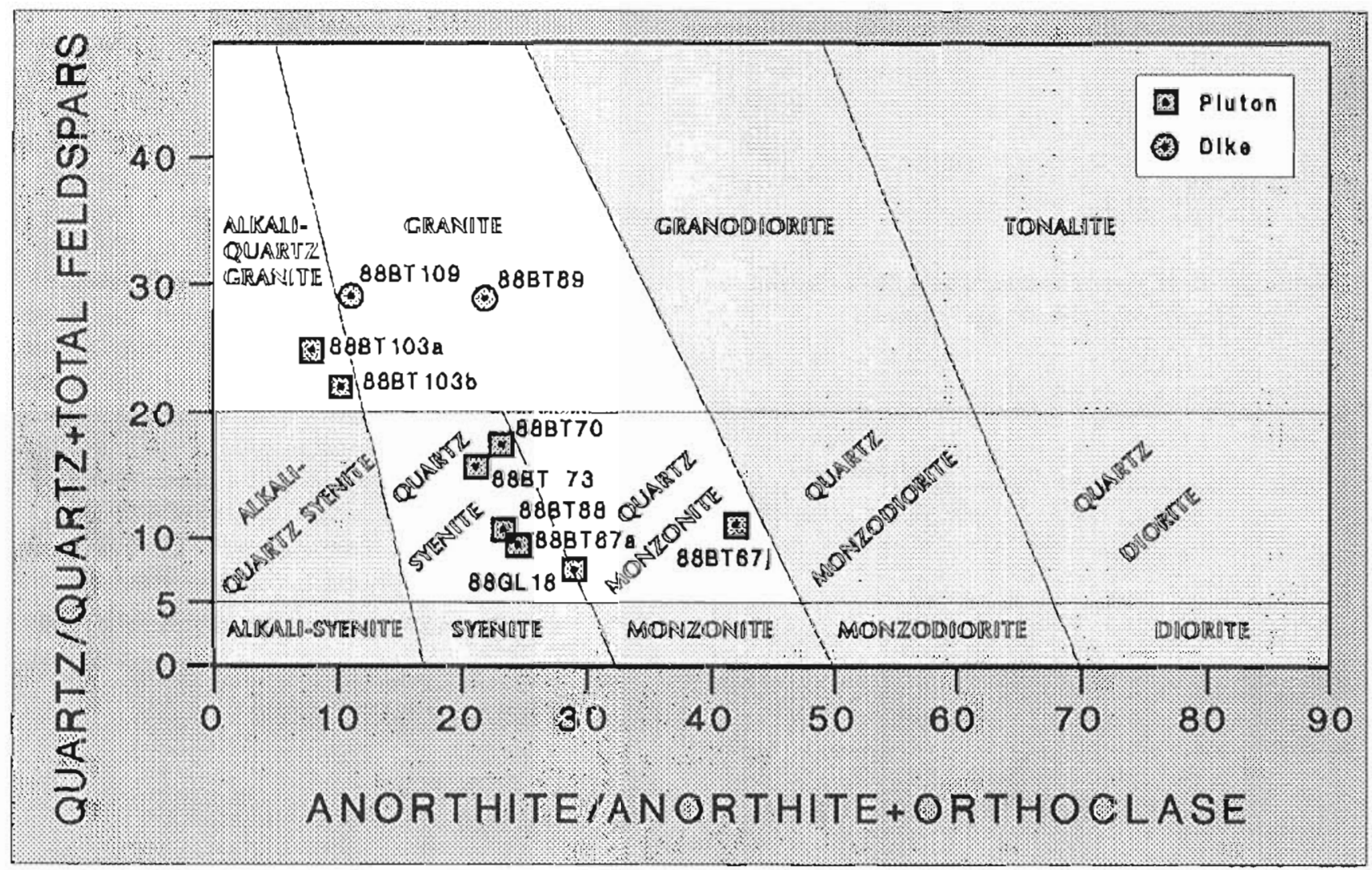

Figure 9. Normative quarlz-K-spar-plagioclase plutanic classification scheme for Late Cretaceous granitic rocks from Russian Mission C-J Quadrangle. Alaska (after Streckeisen and LeMaitre, 1979). Salid. square = pluronic rock: solid circle $=$ dike rock. 
massif. The mapped homfels (TKhf) is composed of a resistant rib of dark-gray, conchoidally fractured, massive metasiltstone-argillite and metasandstone. In thin soction the homfels is easily recognized as recrystallized sandstone and siltstone, but in outcrop it is nearly indistinguishable from aphanitic igneous rock. The metasiltstone-argillite contains white mica, epidote, and rare biotite porphyroblasts; the metasandstone is simply recrystal ized quartz and feldspar. Rare porphyroblasts of andalusite are present in soulheastert exposures of the hornfels within $100 \mathrm{~m}$ (328ft) of the pluton. In general, the hornfels grades from massive aphanite near the pluton contact to dominantly cherly argillite and metasandstone $20010400 \mathrm{~m}(660$ to $1,300 \mathrm{ft})$

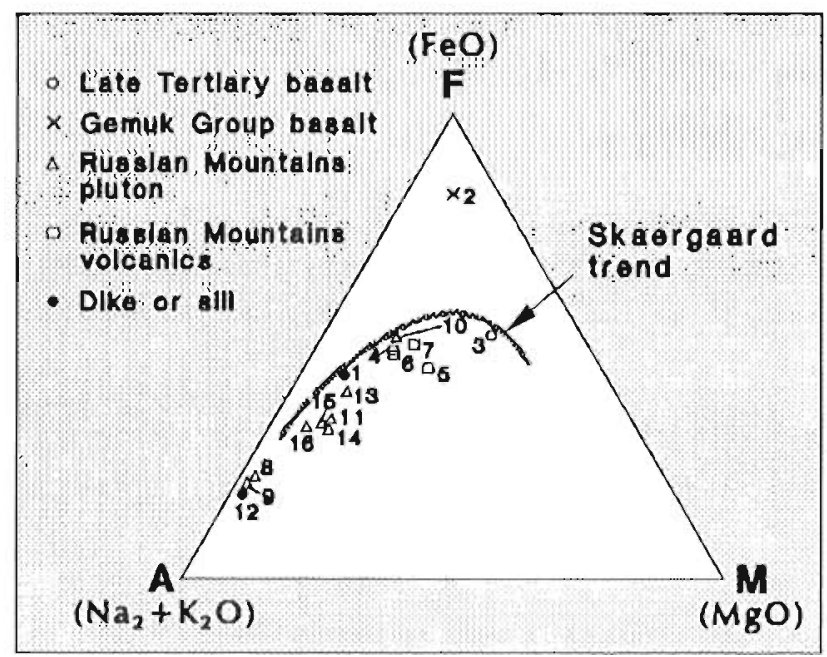

Figure 10. AFM plot of igneons rocks from Russian Mission C-I Quadrangle, Alaska.

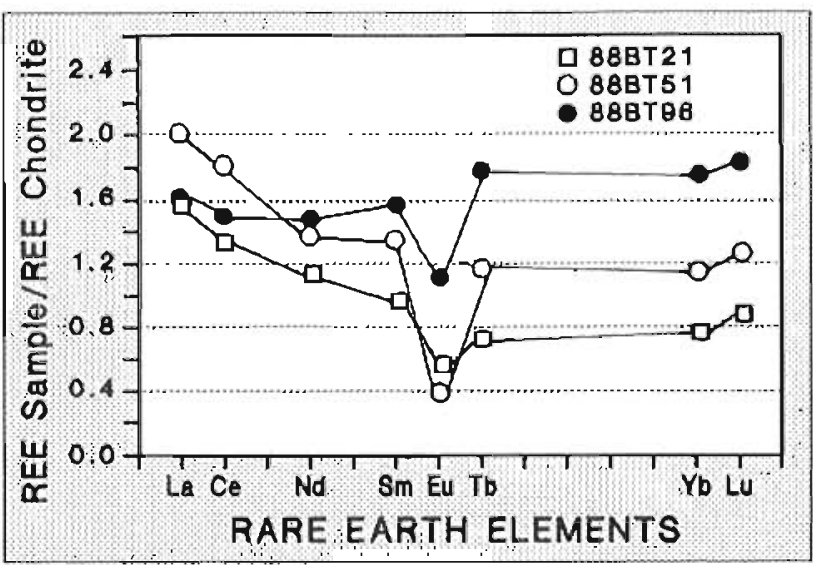

Figure 11. Chondrile-normalizedrare-earthelemen! (REE) concentrations in plutonic rocksfrom Russian Mounrains, Alaska. Analyses by Nuclear Aclivation Services, Onlario, Canada. from the contact to slightly recrystallized sandstone, mudstone, and siltstone 400 to $700 \mathrm{~m}(1,300$ to $2,300 \mathrm{ft})$ from the contace The total width of the thermally disturbed rocks is about $1 \mathrm{~km}(0.6 \mathrm{mi})$.

Overlying volcanic strata have also been thermally affected by the pluton but are not mapped as homfels because in the field the thermally affected areas are indislinguishable from unaffected exposures. However, the volcanic rocks are more reaclive than the metasedimentary racks and therefore result in a more pervasive contactmineral assemblage of biolite, albite, epidote. actinolite, and locally pumpellyite. Locally biotite overprinting is pervasive, resulting in distinct red-brown outcrops and talus that sharply contrast with unaliered gray and purple lavas.

An age of 69.8 Ma (table 1, no. 4) was obtained from andesite hon fels. Thin-section analysis of sample 4 shows apervasive biotitc and sericite overprint of the fabric, which suggests that a thermal event re-equilibrated the $\mathrm{K}^{*}$-A isotopic systcm. The extent of the contact-metamorphosed volcanic rocks could not be determined without additional detailed peorographic studies. Melamorphic conditions in both the volcanic and metasedimentary units are typical of epidote-horn [cts lo homblende-homfels metamorphic facies (Tumer, 1968).

\section{TERTIARY BASALT FLOWS}

Three or four essentially unaltered, vesicular olivineclinopyroxene basalt flows (Tvb) cover a $7-\mathrm{km}^{2}\left(4 \mathrm{mi}^{2}\right)$. elliptical area near the westerm boundary of the map area. The flows are each about $10 \mathrm{~m}$ (32 fi) thick. stighty inclined, and columnar jointed. In a thin section of the basalt, partially antigorized, olivine grains and orthopyroxene are enclosed in a finc-grained, felted groundmass of calcic plagioclase $\left(A n_{63}\right)$ and indeterminate oxides. A major-oxide delermination for the basalt results in a tholeiilic classification aficr schenes sum marized by Irvine and Barager (1971). One whole-rock analysis of vesicular basalt (table 1, no. I) yielded a K-Ar age of $6.19 \mathrm{Ma}$.

The basalı flows straddle and overlie the Kuskok wim Group Flysch and older Gemuk Group greenstone along the Iditarod-Nixon Fork transucrent fault immediately west of the Owhat River.

\section{QUATERNARY DEPOSTTS}

\section{GLACIAL DEPOSITS \\ $\left(\mathrm{Qgt}_{1-1}, \mathrm{Qrg}, \mathrm{Qg}\right)$}

Till ( $\left.\mathrm{QgI}_{1,4}\right)$ in the study area, confinct mainly to the Russian Mountains region, are divided into four distinct subunits based on morphology, lateral extent, and age. These subunits generally correspond to glacial units delin- 


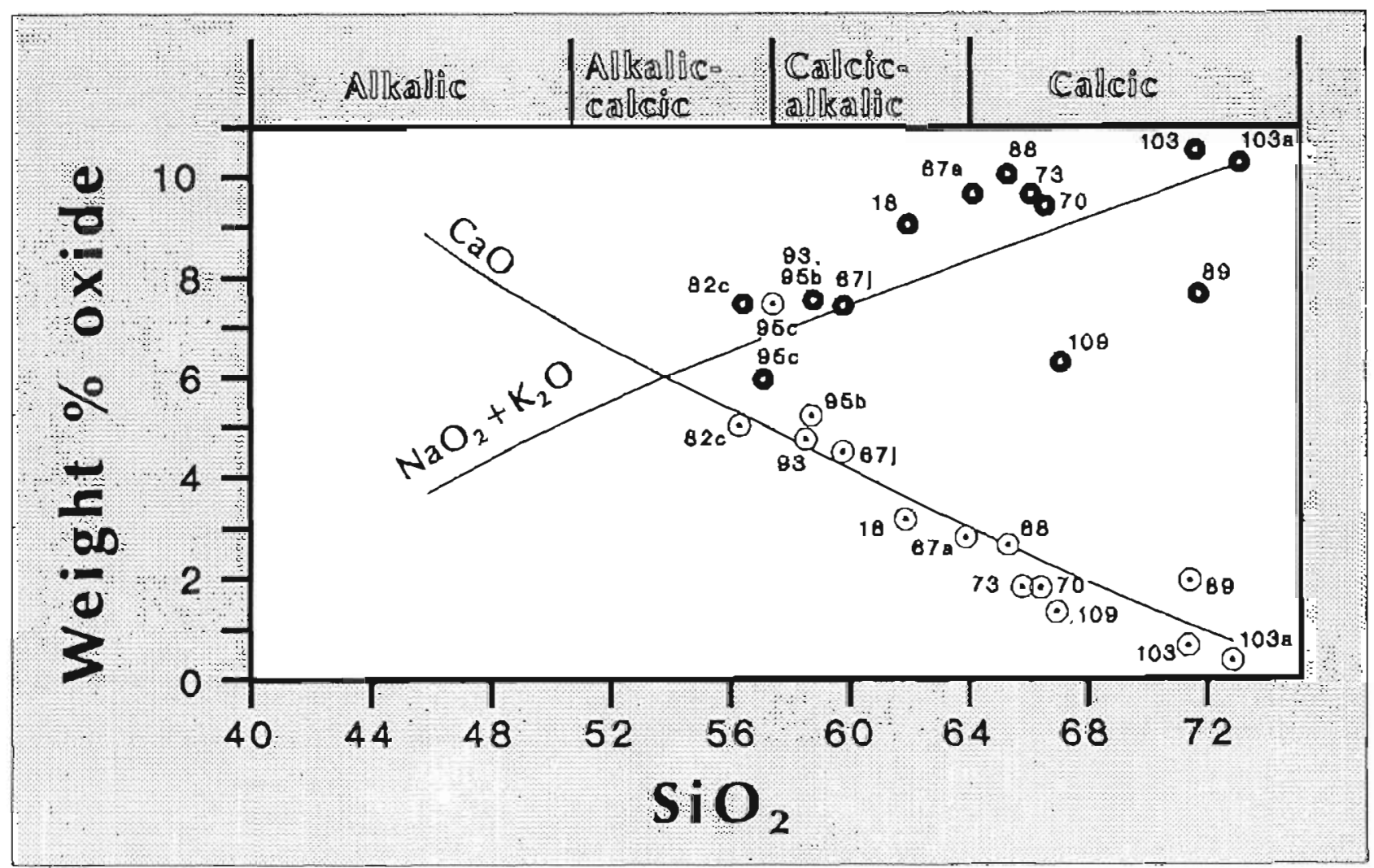

Figure 12. Classification of volcunic and pluonic rocks in Russian Mission C-1 Quadrangle using the alkali-lime index of Peacock (1931). Solid circle $=$ alkali; open circle $=$ linie .

cated by Bundizen (1980) and Kline and Bundizen (1986) in the Kuskokwim Mountiains and the Alaska Range. Becausc no radiometric ages or fossils have been reported from till in the study area, age assignments are based on geologic inference. A summary of glacial advances is shown in figure 13.

The oldest lill $\left(\mathrm{QgI}_{1}\right)$ consists of isolatcd patches of diamicton on two planated, north-facing pediment surfaces northeast of the Russian Mountains (sheel l) at about $400 \mathrm{~m}$ $(1,300 \mathrm{fi})$ clcuation. Isolated plutonic erratics on these surlaces and a series of similar flat rioge crests northwest and southeast of the Russian Mountains (sheet 1) are probably associaled with these deposils. Till exposed on planated summits dissected by erosion is primarily composed of plutonic clasts $10 \leq 20 \mathrm{~cm}$ (8 in.) diam nested in a silt-rich malrix; crosion has crased the glacial-moraine lopography. The oldcse lill correlates wish till of the Beaver Creek glaciation (pre-Wisconsin-probably pre-Illinoian) in the northern Iditarod Quadrangle (Bundizen. 1980). Judging [ron the wide, fan-like configurations of the lill and associated elevaled terrace levels, the Russian Mountains may have supported a small, $310 \mathrm{~km}^{2}\left(120 \mathrm{mi}^{2}\right)$ ice cap during Beaver Creck lime.
The next youngest till ( $\left(\mathrm{QI}_{2}\right)$ appears as distinct terminal and recessional moraines on Mission and Cobalt Creeks and on the east fork of Owhat River and as less extensive moraines on seven unnamed streams that originate in the Russian Mountains. During the glaciation in which this till was deposited, ice occupied al least 24 cirques and all drainages contained some ice (Kline and Bundizen, 1986. p. 147). The targesi trunk glacier occupied the Coball Creek trainage and was fed by (wo main tributary forks to the soulh. It measured almost $12 \mathrm{~km}(7.2 \mathrm{mi})$ long and up 10 $2 \mathrm{~km}$ ( $1.2 \mathrm{mi}$ ) wide during glacial maximums. This till is esscntially identical to that of the Bifurcation Lake glaciation of early Wisconsin age in the Bcaver Mountains (Bundizen, 1980).

Suill younger till (QgL) appears as several arcuate. steep-fronted terminal moraines at the headwaters of Cobalt Creek and the east fork of Owhat River, these deposits mark a maximum ice advance of $3 \mathrm{~km}(1.8 \mathrm{mi})$ long. Unit $\mathrm{Qg}{ }_{3}$ is also present in at lease 13 norh-oriented cirqucs, but absent from sou(h-and west-lacing valleys and cirques. In contrast to the well-dissceled moraines of the next older till unit $\left(\mathrm{QgI}_{2}\right)$. unit $\mathrm{Qgt}$, is largely undissected by stream erosion. We conclate this till with the late Wisconsin 
Figure 13. Map showing extent of Quaternary glacial advances in Russian Mounlains. Alaska. Modified from Kline and Bundizen (/986).

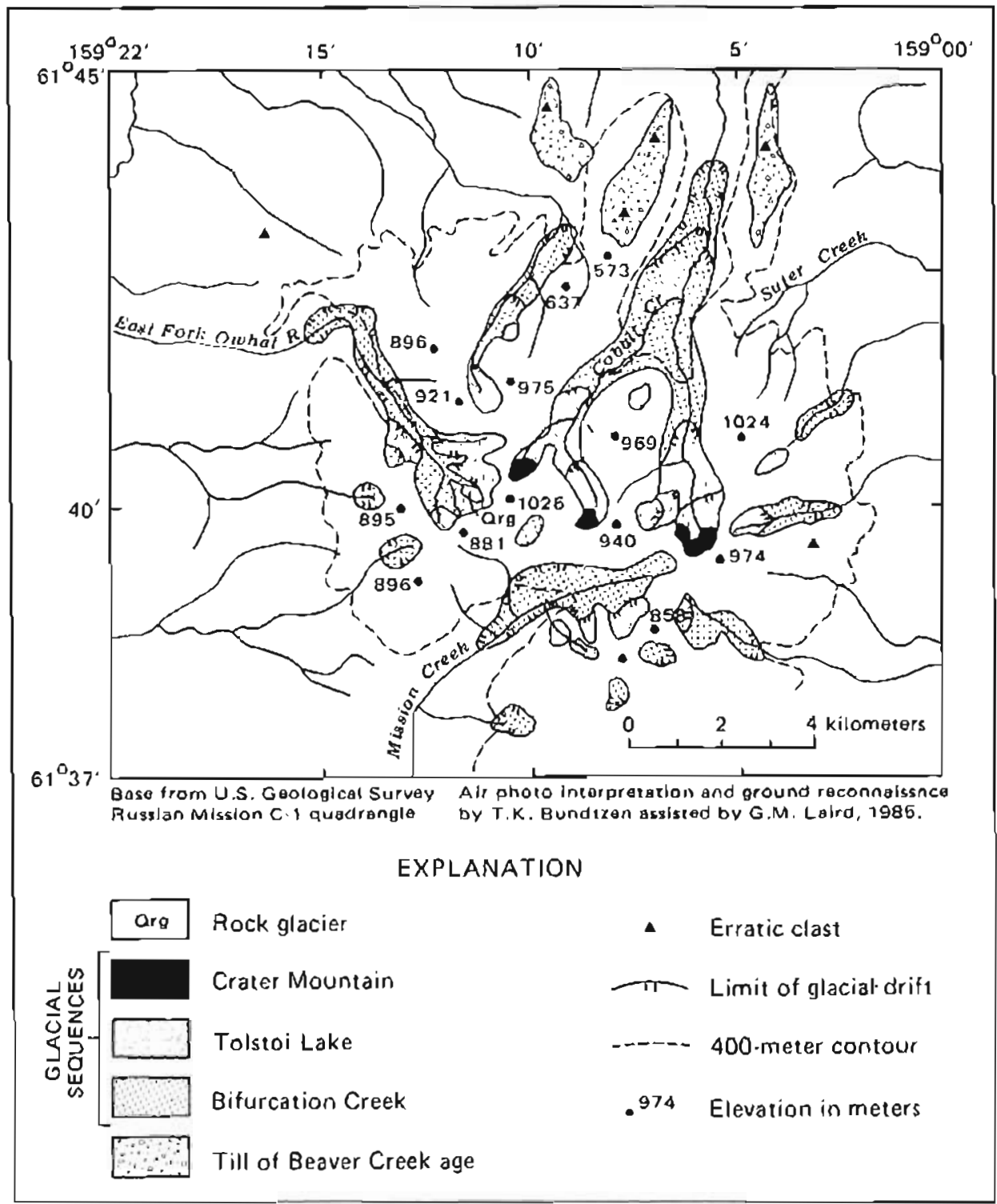

Tolstoi Lake glaciation in the northern Iditarod Quadrangle (Bundtzen, 1980).

The youngest till (Qgt) is confined 10 two or three unusually high north-facing cirques at the extreme head wall of the Cobali Creek drainage. Twenty-four cirques in the Russian Mountains average $610 \mathrm{~m}$ (2.000 fl) elevation: the Holocene(?) cirques average $690 \mathrm{~m}$ (2.265 fl) elevalion. Unit Qgt, is essentially unmodified by stream erosion, and cirque headwalls exhibit little colluvial modification. No soil is developed on Qgt, deposits. Qgt, deposits may be correlative with similar deposits of presumed Holocene age in the Beaver Mountains and Alaska Range (Kline and Bundizen, 1986).

Eight dormane rock glaciers (Qrg) occupy cirques in the Russian Mountains. These spalulate-shaped deposits are composed of poorly sorted angular boulders and smaller rock fragments of intrusive origin. In contrast, on the East Fork O what River, a cirque headwall composed completely of more platy volcanic decritus has formed rock glaciers. All rock glaciers probably formed in cirques of Tolstoi Lake (Late Wisconsin) age at alutudes of $55010610 \mathrm{~m}(1,800$ to 2,000 $\mathrm{ft}$ ) immediately following deglaciation.

During an Early or pre-Wisconsin glacial maxima. several large out wash fans (Qg) were deposited along the flanks of the Russian Mountains. This out wash is composed of almost equal amounts of plutonics, volcanics, and hornfels that appear as $20 \mathrm{ft}$ to 150 -m-thick (65 to $490 \mathrm{ft}$ ) terraces above modern dissected drainages. The largest Qgf deposits on Mission Creek and Easi Fork Owhat River are $5107 \mathrm{~km}$ ( 3 to $4 \mathrm{mi}$ ) long, about $6 \mathrm{~km}(3.6 \mathrm{mi})$ wide, and average about $25 \mathrm{~m}$ ( $82 \mathrm{ft}$ ) thick, based on Kuskokwim River culs of the outwash fan. Similar fan-shaped outwash plains have boen 
reported in the Beaver, Horn, and Chuilnik Mountains (Bundtzen, 1980; Cady and others, 1955; Waythomas, 1984).

\section{COLLUVIAL DEPOSITS (Qca, Qct, Qct)}

Following each successive glacial maxima and ice recession, valley walls collapsed and were rapidly modified by slope processes that built colluvial-alluvial fans $(\mathrm{Q} c \mathrm{ca})$ and colluvial-talus deposits at slope apices $(\mathrm{Qct})$. Along the southern limit of the Russian Mountains, poorly sorted composite alluvial-colluvial aprons of fluvial fans and slope colluvium $(Q c t)$ probably formed in part from the reworking of Earty or pre-Wisconsin glacio-fluvial deposits of the Mission Creek outwash fan (Qgf).

Poorly stratified, organic-rich, bog silt (Qsp) covers glacio-fluvial deposits in the Russian Mountains and along the floodplains of the Kuskokwim and Aniak Rivers in the southem part of the study area. In both instances, Qsp deposition reflects stagnation of the previous higher energy fluvial environment that existed in these areas. Peat deposits (Qsp) in the Russian Mountains consist of well-stratified, frozen, peat layers $2 \mathrm{~cm}$ to $2 \mathrm{~m}$ ( 1 in. to $6.5 \mathrm{ft}$ ) thick in lenticular, mound-like palsas that reach thicknesses of $10 \mathrm{~m}$ (33 ft).

\section{ALLUVIAL DEPOSITS (Qat, Qas, Qag, Qa)}

Older fluvial deposits (Qat) are relict paleostreams and parallel modem drainage pattems. These deposits are composed of limonitically stained clasts of igncous and sedimentary rocks from local sources. Most deposits are presumably of Quaternary age, but high-level deposits may be Late Tertiary terraces. Sandy to cobble-sized floodplain alluvium (Qa, Qag) continues to be deposited in rivers and streams in the study area. The alluvium in upland areas is mainly coarse cobble-sand-silt derived from local bedrocks (Qag). Fluvial deposits in the Kuskokwim River valley are finer grained sand-silt-clay in part derived from bedrock and glacial debris that originated from upstream sources (Qa). During seasonal flooding, overbank muds up to $1 \mathrm{~m}$ ( $3.3 \mathrm{ft}$ ) thick cap floodplain deposits up to $3 \mathrm{~m}$ ( $10 \mathrm{ft}$ ) above normal high water levels; mappable overbank deposits are depicted as Qas.

\section{STRUCTURE}

Pre-Cretaceous layered rocks have undergone at least two episodes of northeast-trending, subisoclinal to open folding (sheet 1 , cross sections A, B). The comparative nature and timing of the fold deformation between the
Gemuk Group and the Kuskokwim Group were not accurately determined during this study. In outcrop, axes of $F_{1}$ folds trend from N. $10^{\circ}$ E. to N. $60^{\circ}$ E. and plunge steeply to the northeast. Locally, secondary low-angle cleavage has cut the folds, suggesting significantlow-angle compressional stress in the layered rocks. Bedding attitudes in the Kuskokwim Group appear to all be right-side-up in the sudy area, but overturned beds were observed by Bundtzen and others (1989) at Gibraltar Point about $20 \mathrm{~km}(12 \mathrm{mi})$ upriver in the Sleetmute Quadrangle. The timing of most fold deformation in the MzPzus, Kus, and Kls units probably coincides with Late Cretaceous compressional tectonic activity documented in the McGrath, Lime Hills, and Iditarod Quadrangles north of the study area (Bundtzen and Gilbert, 1983).

The Upper Cretaceous andesitic lavas and tuffs in the Russian Mountains have been warped into broad, northsouth trending synclines, which suggests that a mild compressional episode followed Late Cretaceous-early Tertiary igneous activity.

Several prominent, N, $25-30^{\circ} \mathrm{W}$.-trending vertical faults cut the Russian Mountains volcanic-plutonic complex in Cobalt Creek and Enst Fork Owhat River. These fractures parallel a prominent joint orientation mapped throughout the pluton (sheet 1). Hydrothermal fluids and polymetallic sulfide mineralization have bled in to the northwest-trending fractures-probably shorly after crystallization of the pluton-and created the mappable sericitc-ankerite alteration zones described briefly in the Economic Geology section of this report. In the valley of East Fork Owhat River, syenomonzonite appears to be juxtaposed against porphyritic quartz monzonite along a N. $20^{\circ} \mathrm{W}$.-trending fault.

Curvilinear high angle faults juxtapose andesitic lavas against quartz monzonite in the eastenn Russian Mountains (sheet 1). Previous workers (Cady and ol hers, 1955) suggest that this contact relationship represents caldera collapse into a rising magma chamber, however, this circular fault relationship is not typical of most volcanic-plutonic contacts in the Russian Mountains.

The north-northeast-trending high-angle "Owhat fault" juxtaposes the Kuskokwim Group against older Gemuk Group lithologies west of Owhat River (sheet I). Movement along the "Owhat fault"-probably a splay of the Iditarod-Nixon Fork fault (INF)-appears to predate a $6.2 \mathrm{Ma}$ old basaltic flow section (Tvb) previously described. However, lower Quaternary terrace deposits in the Moore Creek area about $100 \mathrm{~km}(62 \mathrm{mi})$ northeast of the study area may be of fset by the INF fault (Bundtzen and others, 1988a).

Miller and Bundtzen (1988) proposed a Late Cretaceous to Present right-lateral offset solution of $881094 \mathrm{~km}$ (53 to $56 \mathrm{mi}$ ) for the INF fault based on an offset restoration 
of the Upper Cretaceous Iditarod volcanic complex in the Beaver Mountains north west of the fault with the same unit in the DeCourcy Mountain area southeast of the fault. This offset solution places the Russian Mountain complex (in Late Crelaceous time) direculy south of similar but more mafic volcanic-plutonic complexes at Flat in the Iditarod mining district.

\section{ECONOMIC GEOLOGY}

\section{INTRODUCTION}

Mineralization in the Russian Mission C.1 Quadrangle consise mainly of several intrusive-hosted. coppergold-arsenic deposits that contain anomalous amounis of antimony, lin, zinc, bismuth, lead, lungsten, uranium, and cobalt. 'Two deposits have been explored with pits, shafts, and drifts, but no commercial produclion has been recorded (sheet 2). Heavy-mineral placer deposits have been idenufied in immature high-energy glacial outwash on Cobalt and Mission Creeks, but no gold production is known. Modcst amounts of sand and gravel have been mined for local projects. and riprap prospecis have been recenlly identified in the Russian Mountains and along the Kuskokwim River.

\section{LODE DEPOSITS}

\section{OWHAT PROSPECT}

The Owhat quarlz-gold-sulfide deposit was first discovered by Native prospectors near the head of Cobalt Creek (sheets 1,2) shortly before 1900 (Cady and others, 1955: Holzheimer, 1926). Upon receiving information from the discoverers, Gordon Betues prospected the Owhat property for gold shortly after 1900. Maddren (1915. p. 359360) reponed that by 1914 , four claims of the "February Group" covered about $1,220 \mathrm{~m}(4,000 \mathrm{ft})$ of strike length along a "vein deposit of the fissure type." Early prospectors drovea $12 \mathrm{~m}$ (39ft) shaft to explore the deposit. Holzheimer (1926) showed ithree shafts (the number observed during our investigations) on the vein along the eastern edge of the "Mary" and "Louise" claims.

The Owhat Prospect occurs in a bedrock lip that separates Iwo distinct cirque levels at the head of Cobalt Creek [average elevation $480 \mathrm{~m}(1.570 \mathrm{ft})$ ] (sheets 1.2 ). Sulfide-bearing veinsstrike N. $24.28^{\circ} \mathrm{W}$. dip $75-83^{\circ} \mathrm{NE}$., and can be traced for $265 \mathrm{~m}$ ( $870 \mathrm{ft}$ ) before disappearing under talus and glacial till. Isolated mineralized float found along strike to the nortbwest and southeast suggests that the zone runs additional $200 \mathrm{~m}(650 \mathrm{ft})$ in both directions.

The mineralized zone consists of 8 to 10 , closely spaced sulfide-tourmaline-axinile-quartz "greisens." each several centimeters to $1 \mathrm{~m}$ (3.3 $\mathrm{ft}$ ) thick over widths that range from 1.5 to $8 \mathrm{~m}$ ( 5 to $26 \mathrm{ft}$ ); the total griesen zone averages about $2.5 \mathrm{~m} \mathrm{(8ft)} \mathrm{thick.} \mathrm{Near} \mathrm{the} \mathrm{norlhwest} \mathrm{end}$ of the zone. the vein-fault splays into two distincl zones that are separated by host syeno-monzonite country rock. The mineralization is exposed from its lower northwest end at $442 \mathrm{~m}(1,450 \mathrm{ft})$ elevation 10 its higher upper end at $527 \mathrm{~m}$ $(1.730 \mathrm{ft})$ elevation for a total vertical extent of $86 \mathrm{~m}$ (280 ft). Axinile-bearing andesite porphyry (TKdi) makes up most of the eastern hanging wall of the orebody, while syeno-monzonite comprises all of the westem footwall and some of the eastem hanging wall.

Mineral species in the vein include major arsenopyrite, chalcopyrite and arsenopyrite, and pyrite and minor bormite. galena, sphalerite, tetrahedrite, and marcasite in a gangue of quart, lourmaline, and axinite. Microprobe analyses have identified stephanite (silver-antimony sulfide), native bismuth, silver-free tetrahedrite, pekoite or gladite (lead-bismuth sulfide), free gold, aramayoite (silver-bismuth-antimony sulfide), bismuthite, and stetefeldite (silver-antimony hydroxide) (figs. 14,15). Ore textures and field evidence indicate that the intrusive-hosted polymetallic deposits formed during multiple events in the fracture system.

The earliest mineralizing event dislinclly banded, fine-grained, black schorl (lourmaline) that is frequently at lached to the hanging-wall andesite porphyry. The tourmaline bands (orgreisen) contain small xenoliths of syenitemonzonite plutonic rock. The dislinctly red-stainod, euhedral quartz crystals that formed along the foolwall side of the tourmaline greisen during the second mineralizing event have no associated sulfides or free gold.

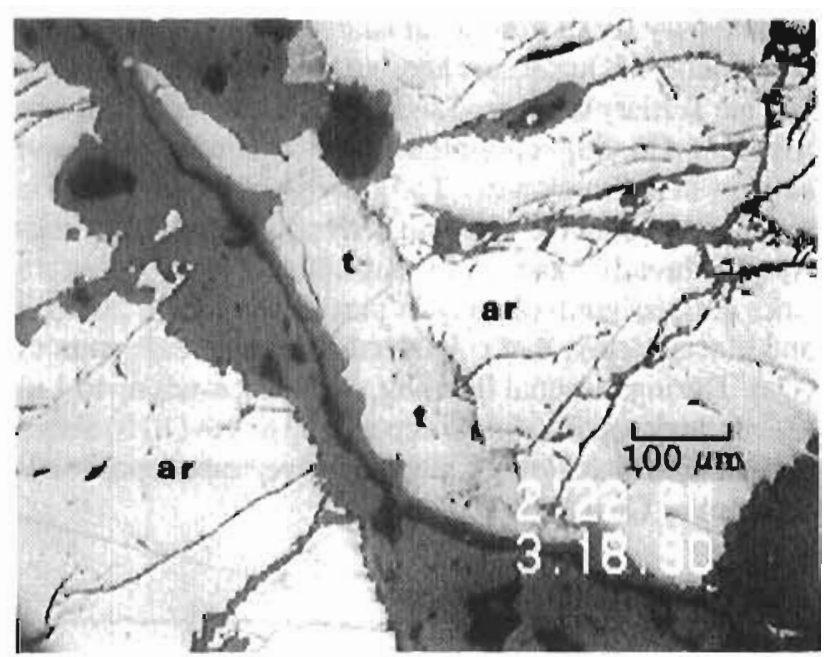

Figure 14.Photomicrograph of silver-free tetrahedrile (l) along fractures in arsenopyrile (ar) from Owhat Prospeci(88BT67u),crossed nicols. Pholographby Cannon Microprobe, Inc. 


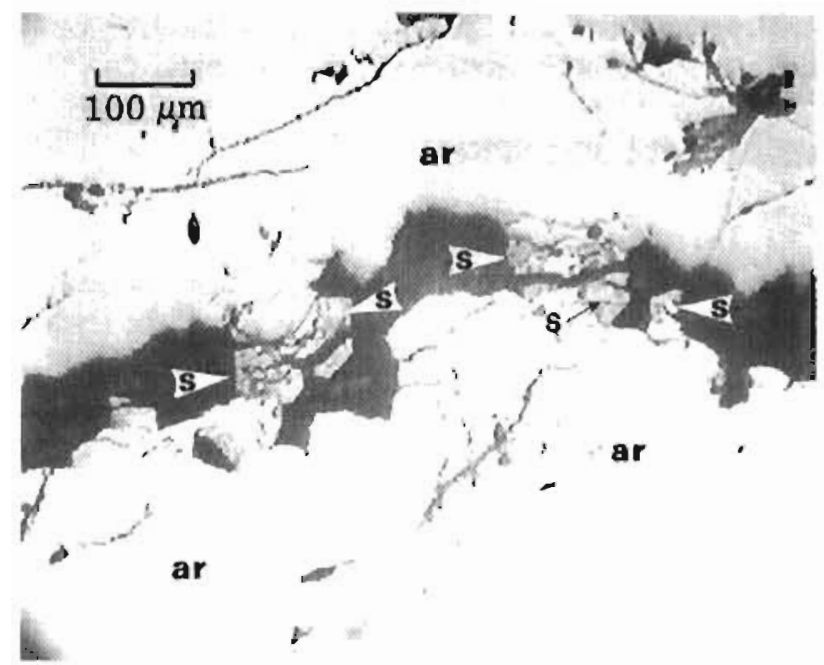

Figure 15. Photomicrograph of stephanile (silver-antimony sulfosali) (s) along fracture in arsenopyrite (ar) from Owhal Prospect (88B7671), crossed nicols. Photograph by Cannon Microprobe, Inc.

A third mineral stage introduced base-metal sulfides (chalcopyrite, auriferous arsenopyrite, and pyrite) and cassitcrite, al replacing vugs and sheets of replaced tourmaline greisen and quarz. Pyrite distinctly infills interstices between euhedral, socond-stage quartz crystals. Some chabcopyrite forms finely laminated bands in the lourmaline-rich cones. A mineralizing [ourh stage introduced bomite, stephanite, tetrahedrite, sphalerite, and tead-bismuth sulfides that clearly crosscul previous sulfides (figs. 14, 15). A filth and linal stage of ore fomation introduced minor arsenopyrite subsequent to brecciation and lault movement along the Owhat structure.

Holzhcimer (1926) reported that "shree 40-rt-deep $(13 \mathrm{~m})$ shafts sunk on massive arsenopyrite kidneys botlomed out in gold-barren chalcopyrite." However, we noled no obvious vertical termination of arsenopyrite mineralizaLion in the 90 vertical meters (295 fl) of the system that we examined. Moreover, microprote analyses indicate that gold occurs as at least two distinct phases at the Owhat Prospect: As blcbs of 900-10-920 fine free gold and bismuthinite in bolh chalcopyritc and arsenopyritc (fig. 16) and as local lattice contam inalion in arsenopyrite. The latter mode of occurrence was determined by comparing "count rales" for gold (Ma) in a standard gold-free arsenopyrite with arsenopyrite from the Owhat Prospect. The results indicate that arsenopyrite from the Owhat Prospect contains 0.1100 .2 weight percent gold in lattice structures.

Bccause no cobalt minerals were identified, tonce analysis using the same prospecting method as that used for gold was also performed on two Owhat Prospect sanples.

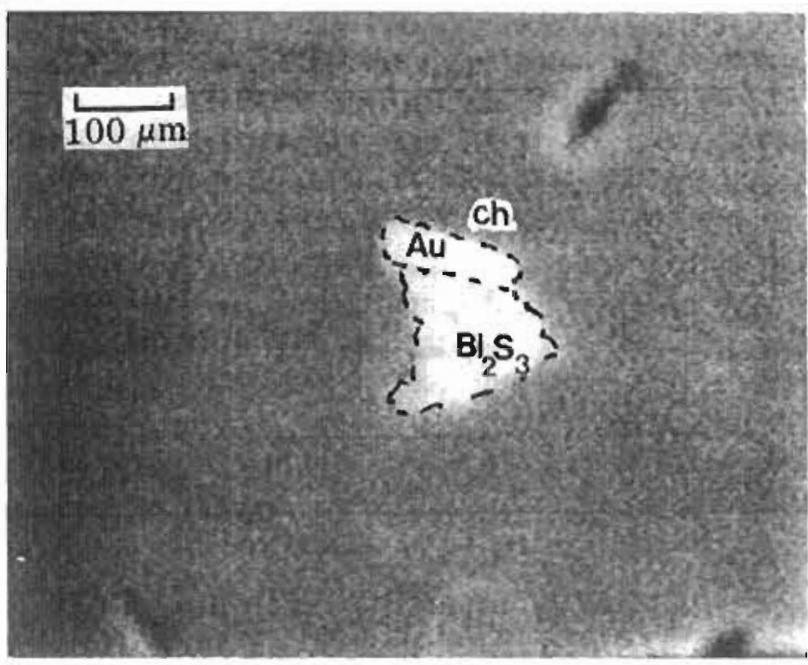

Figure 16. Phoromicrograph of 920 fine gold (Au) and bismulhinite $\left(B i_{2} S_{1}\right.$ ) in chalcopyrite (ch) from $O$ what Prospecl (88BT671), crossed nicols. Pholograph by Cannon Microprobe, Inc.

Results show I 2 to 3.4 percent cobalt in arsenopyrite lallice structures (fig. 17).

We collecled 16 chip-channel samples over an average widih of $1.4 \mathrm{~m}(4.4 \mathrm{ft})$ and a cotal strike length of $262 \mathrm{~m}$ (860 fi). All samples were analyzed for 22 elemenis, but some samples were not analyzed for copper, lead. Iungsten. and lin; unfortunately none were analyzed for bismuth

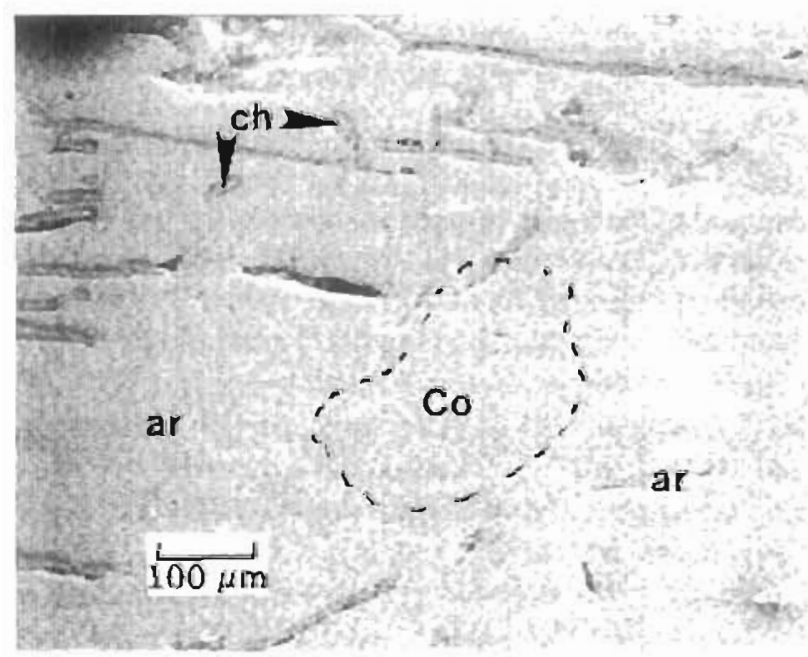

Figure 17. Pholomicrograph of arsenopyrite (ar) that conlains zone of 3.4 percent cobalt (Co). Secondary chalcopyrile (ch) intrudes fractures. Samples from Owhat Prospeci (88BT671), crossed nicols. Phorograph by Cannon Microprobe, Inc. 
(table 3). Holzheimer (1926) provided copper. gold, and silver analyses of channel samples; these analyses are also keyed to sample locations in our sludy (sheet 2).

Anomalous gold was detected in all but one submitted sample; with values range from $380 \mathrm{ppb}(0.012 \mathrm{oz} /$ ton $)$ to $19 \mathrm{ppm}(0.41 \mathrm{oz} / \mathrm{ton})$ gold. Thirteen of 16 samples contained anomalous antimony, four contained anomalous tin. and three contained anomalous tungsten. All samples contained anomalous lead and copper. High gold values clearly follow increases in the arsenic content of the ores at a remarkably consistent ratio of $390 \mathrm{ppb}$ gold for every 1 percent arsenic.

The following reserve calculations assume that the assay data from our 16 samples and from those reported by Holzheimer (1926) are representative of the mineralized system. We also assume that $0.25 \mathrm{~m}^{3}\left(8.5 \mathrm{ft}^{3}\right)$ of mineralization weighs 0.907 metric ton (1 short ton). Two reserve estimates can be calculated: (I) a ninimum inferred reserve based on the volume calculation of a triangular-shaped block of ore defined by known strike length, average width, and verlical extent of mineralization observed on the surface; and (2) a 'half square' estimate that assumes the vertical extent of mineratization is equal to half the known strike length of the are body along segments of the deposit (Harding, 1923: Patterson, 1959). Using an average width

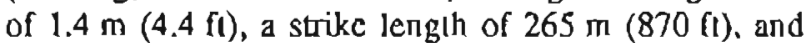
vertical control of $86 \mathrm{~m}$ ( $280 \mathrm{ft}$ ), the Owhat Prospect contains an inferred reserve of 57.000 melric tons (63.000 short tons) that grade $5.3 \mathrm{ppm}$ gold $(0.156 \mathrm{oz} / 10 \mathrm{n}$ gold). 13.4 percent arsenic, 0.21 percent antimony 0.39 percentcopper, 0.07 percent tin, 0.05 percent zinc, and 0.017 percent cobalt. The Owhat deposit contains a half-square reserve estimate of 120,200 metric tons (133.000 short tons) of similar grade.

\section{LOULSE PROSPECT}

The Louise Prospect was first reported by Holzheimer (1926) as a 320-m-long ( $1,000 \mathrm{ft})$ arsenic-gold deposit exposed in the lower cirque basin west of the lowest shafi at the main Owhat Prospect. The old "Louise" claim also includes shafts sunk on the Owhat Prospect. The prospect contains arsenopyrite, chalcopyrite. quartz, and black tourmaline in a poorly exposed vein that trends N. $25-35^{\circ} \mathrm{W}$. for at least $80 \mathrm{~m}(245 \mathrm{ft}$ ) along strike; no vein widths could beestimated, and the occurrence was not sketched As al the Owhat Prospect, altered andesite porphyry dike rock apparently forms part of the hanging wall of the vein; the remaining vein is hosted in syenite. One grab sample from the Louise Prospect (table 3. no. 10. 88BT68) contained 4.1 ppm gold ( 0.12 oz/10n gold), 10 percenl arsenic, 0.99 percent antimony, and 0.12 percent tin. This sample contains $410 \mathrm{ppb}$ gold per 1 percent arsenic-a ratio in remarkable agreement with results from the Owhat Prospect. Cady and others (1955) reported that "two samples from a short shart
$1,000 \mathrm{ft}$ west of the Owhat Prospect are reported to assay 1.40 percent and 1.22 percent in respectively:" these samples are probably from the Louise Prospect.

\section{HEADWALL PROSPECT}

The herein named Headwall) Prospect (table 3, no. 14, and sheets 1,2 ) is located along (he stcep western headwall of a cirque basin about $1 \mathrm{~km}(0.6 \mathrm{mi})$ south of the Owhat Prospect. Although we found the remains of toots and shovels, there are no published references for the deposit. The Headwall Prospect consists of a wide tourmalinequartz-sulfide greisen that crends N. $32-34^{\circ} \mathrm{W}$. and dips steeply south west with a mininum strike of $240 \mathrm{~m}$ (790 ft). The north west exiension of the zone disappears under talus and till in a glacial cirque, but may continue southeast for an unknown distance into the cliff face of the cirque headwall.

Sulfide mineralization and paragenesis are similar to that of the O what and Louise deposits but more disseminated in nature. Several, $3 \mathrm{~m}$-thick (10 ft) banded tourmaline greisen zones are well developed in southeast exposures and are partly replaced by euhedral quartz and sericite. Arsenopyrite. pyrite, and minor chalcopyrite cut the quartz and tourmaline, and xenoliths of the former gangue minerals are nested in sulfides. Additionally, native bismuth. pekoite (lead-bismuth sulfide). galena, bindheimite (antimony hydroxide), and scorodite were identified in microprobe analyscs (fig. 18). Post-mineral movement has broken up the sulfide zone, suggesting fault movement subsequent to mineralization. No copper, bismulh, or lead assays are available from the prospoct.

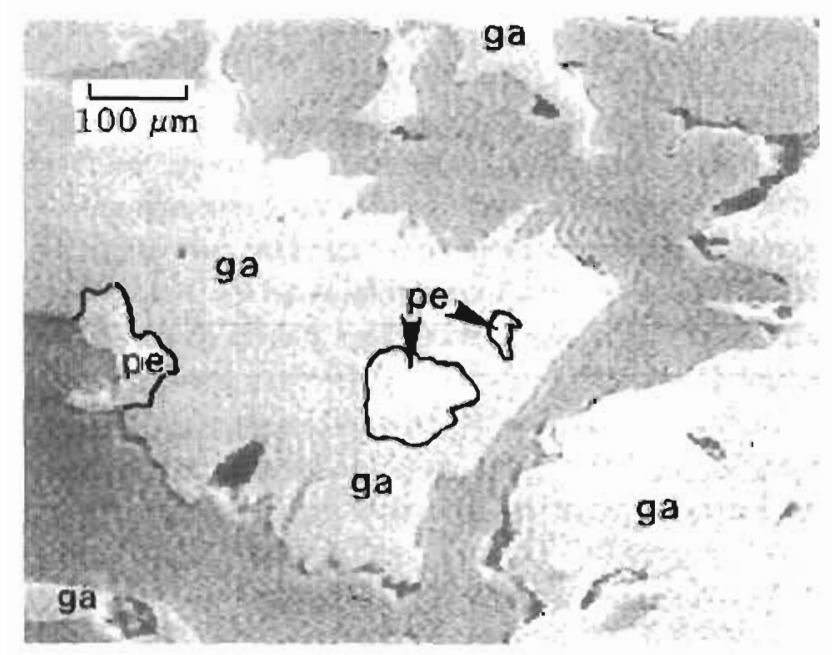

Figure 18. Photomicrograph of pekoite (pe) in galena (ga). Headwall Prospecl (88BT89e), crossed nicols. Pholograph by Cannon Microprobe, Inc. 
Although vertical assay and geologic control of about $120 \mathrm{~m}(400 \mathrm{ft})$ are somewhat uncertain due to the extensive talus that covers more than 40 percent of the strike of the vein, we assume the same assay and geologic criteria calculations as used on the Owhat Prospect Volume and grade calculations use an assay width of $1.36 \mathrm{~m}(4.45 \mathrm{ft})$, a strike length of $240 \mathrm{~m}$ (790 ft), and a vertical extent of

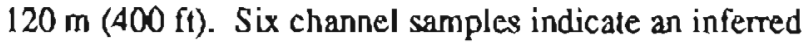
reserve of 75,000 metric tons $(82,900$ short tons) that grade $3.3 \mathrm{ppm}(0.09 \mathrm{oz} / 10 \mathrm{n})$ gold, 7.32 percent arsenic. $0.16 \mathrm{per}-$ cent antimony, and 0.04 percent tin. A "half square" reserve estimate is essentially the same as the surface calculated reserve. Gold values clearly comelate with high arsenic content (450 ppb gold for every 1 percent arsenic), which is similar to results from the $O$ what and Louise deposits.

\section{MISSION CREEK OR "KONECHNEY" PROSPECT}

A complexly mineralized quanz-sulfide-tourmaline greisen vein has been explored by trenching and by two levels of underground drifts at the head of Mission Creek about $1.2 \mathrm{~km}(0.7 \mathrm{mi})$ south of the Headwall Prospect on the soulh side of the Russian Mountains drainage divide (sheets 1, 2). The "Konechney" Prospect, which was described by Holzheimer (1926) and bricfly by Cady and others (1955), has undergone more development than other prospects in the Russian Mountains (fig. 19).

Nine claims were located in 1921 and 1924 by prospectors Joseph Konechney and Charles Warden following their discovery of sulfide float on a low saddle above Mission Creek. Prior to 1926, a 100-m-long (360 ft) drift was driven at the $648-\mathrm{m}(2.150 \mathrm{lt})$ elevation about $60 \mathrm{~m}$ (196 ft) below the discovery outcrops. A skelch of the underground workings from Holzheimer (1926) is included in the mine map on sheet 2. Holzheimer's original sketch refers to a S. $25^{\circ} \mathrm{W}$. strike direction of the vein whereas the true direction is N. $30^{\circ} \mathrm{W}$. Another drift (located about $62 \mathrm{~m}$ (204 fit) vertically below the $648 \mathrm{~m}$ drift) was completed by Konechncy, probably between 1937 and 1942 (Ken Dahl, oral commun., 1988). We were unable to locate underground mapping control from this drift. A rock cabin and blacksmith shop. both in a state of disrepair, were still visible at the $595 \mathrm{~m}(1,950 \mathrm{ft})$ elevation below the mine workings. The portals of both drifts were caved and inaccessible (fig. 19).

The Konechney deposit consists of quartz-sulfidetourmaline greisen veins similar to those observed at the Owhat, Louise, and Headwall prospects. Axinite-bearing andesite-porphyry dike rock parallels the N. $30^{\circ}$ W. average strike of the mineralized zone in the upper levels, but splays off to the north in the lower workings. The zone dips $75-80^{\circ}$ southwest in most surface exposures and in old sketches of underground workings.

Sulfide-gangue paragenesis differs somewhal from the Owhat and Headwall deposits in that chalcopyrite, courmaline, and quartz appear to be introduced simultaneously, followed by arsenopyrite, pyrite, metazeunerile (a copper-uranium-arsenicmineral), free gold, and cassiterile. The cassiterile occurs as small, nearly black crystals interspersed in the quarz-tourmaline gangue on the structure's hanging wall. The last mineral species introduced into the struclure are the low temperature sulfosalts and sulfides [chalcocite, bornite, subnite(?), stephanite and covellite]. Abundant cuprite, azurite, malachite, goethite, and chrysocolla are found on the mine dumps. Wedow (1953) and West (1954) investigated the Konechney deposit because of its anomalous uranium content, and analyses of our

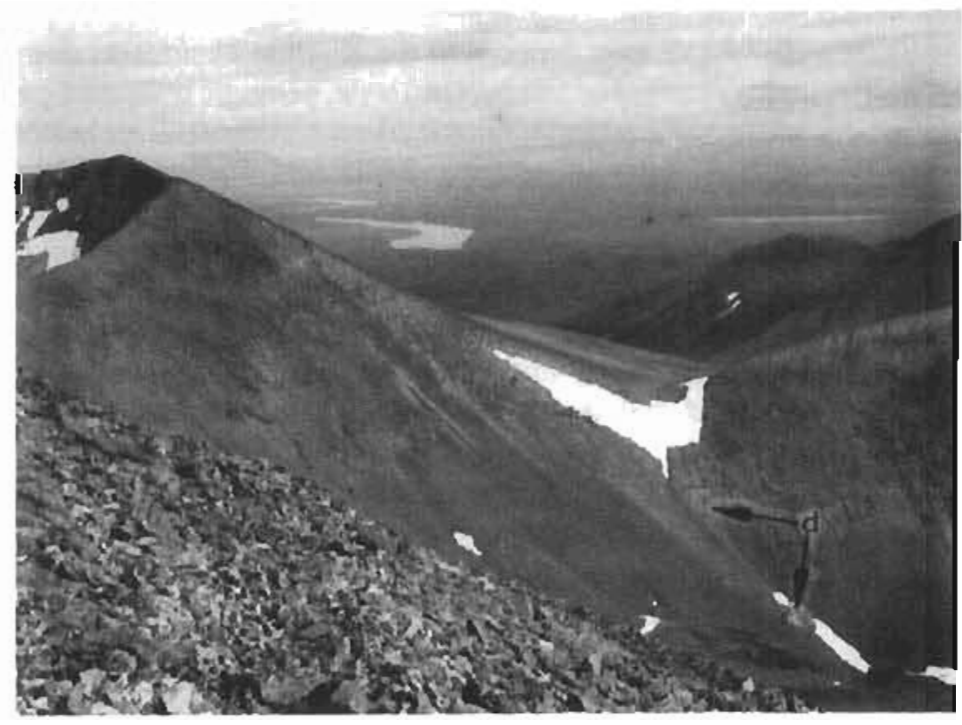

Figure 19. "Konechney" or Mission Creek Prospect area showing mine dumps (d) and discovery area (low saddle); view southeast loward Kuskokwim River. Alaska. 
samples indicate up to $106 \mathrm{ppm}$ uranium (table 3, 85BT184A).

The zone was traced for $150 \mathrm{~m}$ ( $470 \mathrm{ft}$ ) along strike before it disappeared under valley talus and vegetation on its northwest limit. The mineralized zone is truncated on its southeast limit as trenching and sampling failed to locate it beyond the high saddle.

To infer reserves, we used geologic and geometric assumptions similar to those employed on the Headwall and Owhat deposits. Assuming an average assay width of $1.05 \mathrm{~m}$ (3.4 ft), a minimum vertical extent of $120 \mathrm{~m}(400 \mathrm{ft})$, and a strike length of $150 \mathrm{~m}(470 \mathrm{ft})$, the 12 channel samples we collected and five channels samples collected by Holzheimer (1926) suggest an inferred reserve of 34,000 metric tons (37,600 short tons) that average $4.44 \mathrm{ppm}$ $(0.13 \mathrm{oz} / \mathrm{ton})$ gold, 1.64 percent copper, 1.14 percent arsenic, 0.24 percent antimony, with anomalous levels of tin, cobalt, uranium, and silver. One sample (85BT 184D) contained $112 \mathrm{ppm}$ Bi-the only one analyzed for that element. Holzheimer (1926) reported up to $317 \mathrm{ppm}$ ( 9.3 ozton) silver, our maximum analysis for silver is $182 \mathrm{ppm}$ ( $5.3 \mathrm{oz} / \mathrm{ton})$. Volume escimates using the "half square' method understate vertical dimension and overall tonnage based on the surface calculations.

Evidence of a gold-arsenic association in the Konechney deposit is less convincing than the association shown at the Headwall and Owhat deposits. Examination of limited undergtound assay data from Holzheimer (1926) might suggest a gold-copper as well as a arsertic-gold correlation as documented in microprobe analyses of samples from the Owhat Prospect. If gold is only associated with arsenic, then there are $1,140 \mathrm{ppb}$ gold for cvery 1 percent arsenic, which is about three times the ratio calculated for the other deposits.

\section{OTHER LODE PROSPECTS IN THE RUSSIAN MOUNTAINS}

Isolated and generally poorly exposed quartz-sulfide occurrences were noted during our work in the Russian Mountains. Gossan-rich quartz veins occur in N. $30^{\circ}$ W.trending fractures and joints in quartz syenite about $2.5 \mathrm{~km}$ (1.5 mi) southcast of the Konechney deposit (table 3 . nos. 16,17 , and sheet 1 ). The two occurrences, each about $0.5 \mathrm{~m}(1.5 \mathrm{ft})$ wide and $150 \mathrm{~m}(490 \mathrm{ft})$ apart. contain up to 0.30 percent arsenic, 1.50 percent antimony, and $353 \mathrm{ppm}$ neodymium. No sulfides were recognized in the oxidized zones. The dimensions of the occurrence are severely obscured by rubble and vegetation, but both may be associated with nearby andesite porphyry dikes. The occurrences project northwesterly along strike toward the Konechney deposit.

A small drift (now caved) was found in a gossan-rich quartz vein in homfels within $100 \mathrm{~m}$ ( $330 \mathrm{fl}$ ) of the honfels/ intrusive contact zone in the southem Russian Mountains (table 3, no. 9, and sheet 1). Euhedral quartz crystals are particularly well developed in vugs in a sheeted vein, but poor exposure prevented measurement of the dimensions of the occurrence. The quartz vein contains anomalous gold $(250 \mathrm{ppb})$, arsenic (250 ppm), and tin (62 ppm).

A poorly exposed amethyst-bearing vein that contains anomalous uranium (38 ppm) accurs at $365 \mathrm{~m}(1,200 \mathrm{ft})$ elevation about $8 \mathrm{~km}$ ( $5 \mathrm{mi}$ ) north of Chuathbaluk (sheet 1 , no. 20). This prospect, which was discovered in 1960 by C. Abruska and N. Philip, has been referred to as the Ptarmigan Prospect (Ken Dahl, oral commun., 1985). Rubble material indicates that the crystals have grown in small vugs in a brecciated quartz-looded zone in hornfels. Amethyst crystals range from 1 to $5 \mathrm{~cm}(0.4$ to $1.8 \mathrm{in}$.) long and is frequently double-terminated in crystal habit. Colors vary from light purple to blue and average a moderate purple. Lack of exposure prevented further examination of the mineralized zone.

A discontinuous sulfide-quartz vein is hosted in the syeno-monzonite intrusive cupola zone immediately below thermally altered andesite flow rocks (TK vm) in the westem Russian Mountaíns (table 3, no. 2, and sheet 1). Vein mineralization appears to be confined to the intrusive host rock and docs not cxtend upward into the homfelsed volcanic cap rocks. Two 1 - to 3-cm-thick (0.4 to 1 in.) veins have intruded along N. $40^{\circ}$ W.-trending joints in the plutonic rocks for a total tracable length of about $20 \mathrm{~m}$ ( $65 \mathrm{ft}$ ). Malachite-stained rubble-crop conlains minor chalcopyrite, arsenopyrite, and galena in quartz gangue. One grab sample contained anomalous arsenic $(0.46$ percent), antimony ( 0.30 percent), 0.94 percent lead, 0.09 percent cadmium, and silver $(45 \mathrm{ppm})$, but no gold. The occur rence. although smatl, is similar to those of the Owhat. Louise, Headwall, and Mission Creek Prospects.

\section{LODE DEPOSIT PARAGENESIS}

Most lode deposits in the Russian Mountains share many similarities in structural style, geologic relationships, and metallic and mineralogic content. Almost all are hosted in N. 20-40 W.-trending joints and fractures in host plutonic rocks and exhibit a ubiquitous association of axinite-bearing andesite porphyry dike rocks in the hanging walls and footwalls of the vein-fault structures. The andesite dikes may have a genetic association with the orebodies or may be intruding along the same structural channels. Deposits in the Russian Mountains are relatively hightemperature, "greisen-like" vein-faults. Fluid inclusion data from quartz in the Mission Creek Prospect $(\mathrm{N}=4)$ indicate formation temperatures that range from 280 to $410^{\circ} \mathrm{C}$. Limited microprobe analyses from the Owhat Prospect show 29.0 to 33.0 atomic percent arsenic in arsenopyrite. Compositional plots using techniques described by Kretschmar and Scott (1976) show arsenic 
crystallization temperatures of about $320^{\circ} \mathrm{C}$. If cassiterite, arsenopyrite, and chalcopyrite formed synchronously at the Mission Creek deposit, then temperatures of formation were probably $\geq 400^{\circ} \mathrm{C}$ for these mineral species. Tetrahedrite, bismuth, silver minerals, and free gold probably formed at lower temperatures.

The mesothermal to hypothermal(?) quartz-sulfide deposits of the Russian Mountains are similar to those of the Bolivian tin-silver belt-specifically deposits in the Cordillera Quimsa Cruz near LaPaz (Lindgren, 1936; Sillitoe and others, 1975). However, the deposits in the Russian Mountains exhibit an uncommon association of gold and tin. In many hydrothermal mineral deposits, the presence of gold usually indicates the absence of anomalous tin and vice versa, and gold is not a noteworthy occurrence in the Bolivian tin deposits (Lindgren, 1936; Cox and Singer. 1986). Similar auriferous deposits rich in copper and arsenic are described in the Beaver Mountains and at Golden Horn Prospect area near Flat (Bundtzen and Laird, 1982; Bull, 1988; Bundtzen and others, 1988b).

Although the deposits in Cobalt and Mission Creeks are modest in size, all four occurrences represent a definable structurally controlled zone of vertically dipping, intrusivehosted ore shoots that strike N. $25-35^{\circ} \mathrm{W}$. and are discontinuously exposed over a strike distance of $5 \mathrm{~km}$ ( $3 \mathrm{mi}$ ) and a strike width of $3 \mathrm{~km}(1.8 \mathrm{mi})$. Limited channel sampling at the four deposits shows a minimum inferred reserve of 229,200 metric tons $(253,500$ short tons) that grade $3.7 \mathrm{ppm}$ $(0.12 \mathrm{oz} /$ ton $)$ gold, 0.19 percent antimony, 9.59 percent arsenic, 0.61 percent copper, 0.014 percent tin wilh anomalous bismuth, silver, cobalh tungsten, thorium, uranium. and zinc. It is probable that a larger resource exists in these mineral zones.

Miller and Bundzzen (1988) suggest that when rightlateral translation along the Iditarod-Nixon Fork Fault is restored, the Russian Mountains align in a north-south configuration with the volcanic-plutonic complexes in the Iditarod mining district. We note similarities between the arsenic-enriched, intrusive-hosted gold deposits in the Russian Mountains and the Golden Horn arsenic-scheelite gold deposits hosted in the Black Creek monzo-gabbro in the Iditarod mining district. However, tourmaline enrichments that typify the Russian Mountains system are generally lacking in the Iditarod district deposits.

Bundzen and Miller (1989) presented a metallogenic model for ore deposits associated with meta-aluminous and peraluminous, alkali-calcic, Late Cretaceous to early Tertiary stocks, volcanics, and sills in the Kuskokwim Mountains. The model classifies polymetallic deposits formed in epithernal to hypothermal conditions by comparing temperature-pressure, mineralogical, alteration, structural, and geological data. These workers suggest that all deposits represent similar vertically zoned systems found in several erosional levels in the Kuskokwim Mountains. Intrusivehosted polymetallic deposits in the Russian and Beaver Mountains and the Black Creek stock are part of the highest temperature-pressure, mesothermal to hypothernal(?) intrusive-hosted ore deposits of the "Kuskokwim mineral belt."

\section{PLACER DEPOSITS}

Despite the presence of gold-bearing lode sources for placer concentrations in streams of the Russian Mountains, there is no record of placer-gold or other heavy-mineral production in the study area. Multiple glaciofluvial cycles may have buried or diluted placer mineral concentrations in the Russian Mountains. Prospectors have searched for but not found economic concentrations of placer gold in the adjacent Owhat Upland (Maddren, 1915).

The two known placer occurrences in the Russian Mountains are described below. According to Maddren (1915, p. 339), "prospects of placer gold are present in the gravels of Mission Creck below the (Konechney) prospect, and the placer gold is presumed to be derived from the mineralized zones." We did not investigate these placer deposits. We panned placer gold in Cobalt Creek about $1.5 \mathrm{~km}(2.5 \mathrm{mi})$ below the Owhat gold-arsenic-copper deposit (table 3, no. 12, and sheet 1); concentrates from three panned samples yielded $68 \mathrm{ppm}(2.0 \mathrm{oz} / \mathrm{ton})$ gold. 0.042 percent arsenic, 0.15 percent tin, and 0.013 percent tungsten. The samples were obtained by extracting fine sands and gravels from a coarse boulder pavement of syenomonzonite float up to $1 \mathrm{~m}(3.3 \mathrm{ft})$ in diam. Hence, despite positive gold values, the nature of the placer deposit could pose significant engineering obstacles.

Bundizen and others (1987) reported anomalous placer gold below dissected till of Early Wisconsin age on Tolstol Creek in the Beaver Mountains about $120 \mathrm{~km}$ (75 mi) north of the Russian Mountains. Both the Beaver and Russian Mountains contain similar hardrock metallic lodes, have similar glacial histories, and lack exploited placer deposits. Early or pre-Wisconsin auriferous till, if reworked by Holocene strearns, might produce economic concentrations of heavy minerals. We suggest that sites below the terminuses of $\mathrm{Qg} \mathrm{L}_{2}$ and $\mathrm{Qgt}$, till on Cobalt and Mission Creek deserve to be prospected for placer gold and other heavy minerals.

\section{INDUSTRIAL MINERALS}

\section{AGGREGATE}

Coarse cobble- or boulder-bearing outwash deposits form large fans on the East Fork Owhat River and in the Mission Creek drainage (sheet 1). The Mission Creek fan includes a multimillion cubic meter aggregate resource 
accessible by the Kuskokwim River. Gravel at the river edge consists of 20 - to $35-\mathrm{m}$-thick (65 to $115 \mathrm{ft}$ ) zones of intertedded coarse cobble gravel and sand containing equal amounts of volcanic and intrusive floal from the Russian Mounlains. Sieve analysis of a $20-\mathrm{kg}(44 \mathrm{lb})$ gravel sample (after selective removal of cobbles), indicates a coarse silty-sandy gravel aggregate. Approximately 35 percent of this material was coarser than $9.5 \mathrm{~m}(3 / 8$ sieve), and nearly 10 percent was coarser than a $37.5 \mathrm{~mm}$ (1-1/2 in.) sieve (fig. 20). In 1987, 14,570 metric tons (16,100 short tons) of outwash from the Mission Creek fan was used in Chuathbaluk for road repairs, airport maintenance, and foundation preparation. More production $\propto c$ curred in 1988 and 1989.

Several hundred thousand tons of channel lag sands and pea gravels in the Kuskokwim River [Birch Tree crossing about $40 \mathrm{~km}$ ( $24 \mathrm{mi}$ ) below Chuathbaluk] have been dredged and shipped to Bethel for construction needs. Similar resources are present beneath the active flood plain and in oxbow bends (Qag) of the Kuskokwim River within the map area, but none (to our knowledge) have been developed (Krause, 1984).

\section{RIPRAP}

Bundtzen and others (1989) assessed three river-front bedrock sites in the study area for riprap. Because the City of Aniak, immediately downstream from the study area in the Russian Mission C-2 Quadrangle, has experienced severe bankerosion during spring flooding and migration of the active channel of the Kuskokwim River, the location of a kcal riprap source is a high prionity.

At the "southcentral Russian Mountain site, 87MDT32" (Bundzen and others, 1989) $8 \mathrm{~km}$ (4.8 mi) upriver from Chuathbaluk, a steep bluff of coarse sandstone, was tested and determ ined to be a submarginal riprap source. At "Chuathbaluk site, 87MDT33," prominent mafic dikes and homfels contain $11,000 \mathrm{~m}^{3}(13,500$ shon tons) Class I and Class II riprap (Bundtzen and others, 1989). However, the site's Alaska T-13 degradation testing value of 10 is below that recommended for riprap.

According to Bundizen and others (1989), the quartz monzonite exposures at the southwest toe of the Russian Mountains contain the best local riprap resources. Almost $2,000,000 \mathrm{~m}^{3}$ ( 6 million short tons) of shallow, near surface Class I-II riprap is accessible from Chuathbaluk $4 \mathrm{~km}$ (2.4 mi) south of the site. A T-13 degradation value of 86 was obtained for the quartz monzonite, the highest quality exhibited by any sample analyzed during the Kuskokwim riprap study. Although the quarz monzonite could be influenced by ground water weathering (Bundzen and others. 1989), we believe that shallow surface reserves alone constitule an adequate resource for riprap projects in the area. Further site evaluation should include drilling and blasting for conformatory testing.

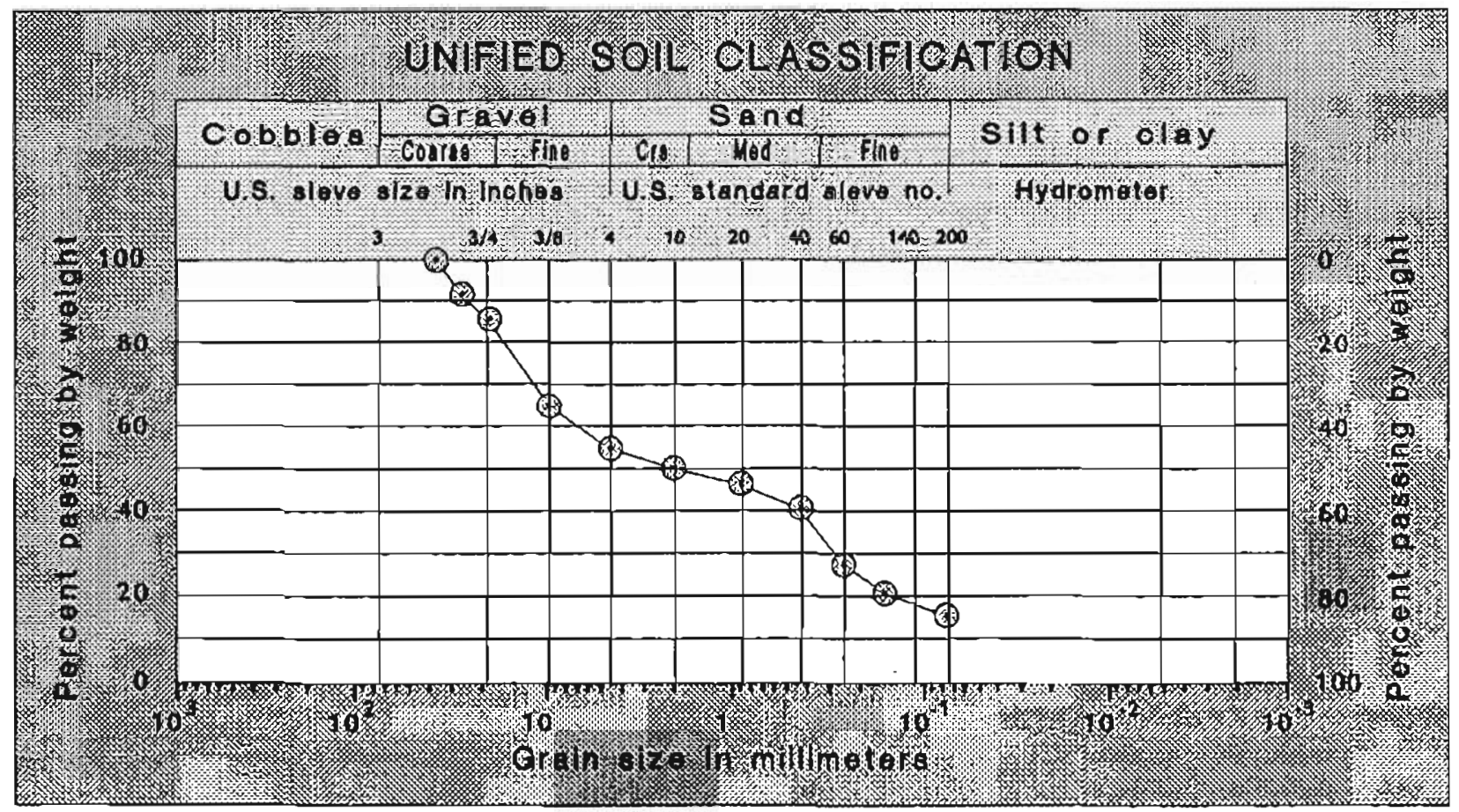

Figure 20. Plot showing grain-sï' distribution of ourwash of Mission Creek fan, Russian Mission C-1 Quadrangle, Alaska. 


\section{REFERENCES}

Alaska Department of Natural Resources, 1986, Kuskokwim area plan, $272 \mathrm{p}$.

Box, S.E., 1983, Tectonic synthesis of Mesozoic histories of the Togiak and Goodnews terranes, southwest Alaska [abs.]: Geological Society of America Abstracts with Programs, v. 15, no. 5, p. 406.

1985, Terrane analysis, northem Bristol Bay region, southwestern Alaska: Development of a Mesozoic intraoceanic arc and its collision with North America: Santa Cruz, University of California, unpublished $P$ h.D. thesis, $163 \mathrm{p}$.

Bull, K.F., 1988, Genesis of the Golden Horn and related mineralization at Flat, Alaska: Fairbanks, University of Alaska, unpublished M.S. thesis, 299 p.

Bundtzen, T.K., 1980, Multiple glaciation in the Beaver Mountains, western interior Alaska: in Larson, Frank, ed., Short notes on Alaskan geology 1979-80: Alaska Division of Geological and Geophysical Surveys Geologic Report 63, p. 11-18.

Bundtzen, T.K., Cox, B.C., and Veach, N.C., 1987, Heavy mineral provenance studies in the Iditarod and Innoko districts, western Alaska, in Vassiloo, A.H., Hausen, D.M., and Carson, D.J., eds., Process mineralogy VII: Warrendale, Pennsylvania, The Metallurgical Society, p. 221-245.

Bundtzen, T.K., and Gilbert, W.G., 1983, Outline of geology and mineral resources of upper Kuskokwim region. Alaska, in Reed. K.M.. ed., Proceedings of the 1982 Symposium on Western Alaska Geology and Resource Polential: Anchorage, Alaska Geological Society, v. 3, p. 101-119.

Bundizen, T.K., and Laird, G.M., 1982, Geologic map of the Iditarod D-2 and eastern D-3 quadrangles, Alaska: Alaska Division of Geological and Geophysical Surveys Geologic Report 72. scale 1:63,360, 1 sheet.

1983, Geologic map of the Iditarod D-1 Quadrangle, Alaska: Alaska Division of Geological and Geophysical Surveys Professional Report 78, scale $63: 300,1$ sheet.

Bundizen, T.K., Laird, G.M., and Gilbert. W.G., 1989, Material studies along Kuskokwim River, McGrath to Kalskag, southwest Alaska: Alaska Division of Geological and Geophysical Surveys Public-data File Report 89-16.84 p.

Bundtzen, T.K, Laird, G.M., and Lockwood, M.S., 1988a, Geologic map of the IditarodC-3 Quadrangle, Alaska: Alaska Division of Geological and Geophysical Surveys Professional Report 96. 13 p., scale 1:63,360, 1 sheet.

Bundizen, T.K., and Miller, M.L., 1989. Geology and metallogeny of Cretaceous-Early Tertiary volcanic and plutonic rocks of western Alaska: Circum Pacific Council on Energy and Mineral Resources Meeting, Kharbarovsk, USSR, 1989, 8 p.

Bundtzen, T.K., Miller, M.L, Bull, K.F., and Laird, G.M, 1988b, Geology and mineral resources of the Iditarod mining district, Iditarod B-4 and eastern B-5 Quadrangles, westcentral Alaska: Alaska Division of Geological and Geophysical Surveys Public-data File Report 88-19, 47 p., scale 1:63,360, 1 sheet.

Bundtzen, T.K., and Swanson, S.E., 1984, Geology and petrology of igneous rocks in the Innoko River area. western Alaska: Geological Society of America Abstract with Programs, Cordilleran Section, v. 16, no. 5 , paper 46990 , p. 273.

Cady, W.M., Wallace, R.E., Hoare, J.M., and Webber, E.J., 1955, The central Kuskokwim region, Alaska: U.S. Geological Survey Professional Paper 268, 132 p.

Cox, D.P., and Singer, D.A., eds., 1986, Mineral deposit models: U.S. Geological Survey Bulletin 1693,379 p.

Decker, John, Robirson, M.S., Murphy, J.M., Reifenstuhl, R.R., and Albanese, M.D., 1984, Geologic map of the Sleetmute A-6 Quadrangle: Alaska Division of Geological and Geophysical Surveys Report of Investigations $84-8$, scale $1: 40,000,1$ sheet.

Harding, J.E., 1923, How to calculate tonnage and grade of an ore body: English Mining Journal, v. 116, p. 445448.

Hietanen, Anna, 1963, Idaho batholith near Pierce and Bungalow: U.S. Geological Survey Professional Paper 344, p. $1-42$.

Hietanen, Anna, and Erd, R.C., 1978, Ferroaxinites from the Feather River area, northern Califomia, and from the McGrath and Russian Mission Quadrangles, Alaska: U.S. Geological Survey Journal of Research, v. 6, p. 603-610.

Hoare, J.M., and Coonrad, WL., 1959, Geology of the Russian Mission Quadrangle, Alaska: U.S. Geological Survey Geologic Investigations Map 1-292, scale $1: 250,000,1$ sheet.

Holzheimer, F.W., 1926, Lode prospects in the Russian Mountains: Alaska Territorial Department of Mines MR 81-1, 13 p. (available at Division of Geological and Geophysical Surveys, Fairbanks, Alaska).

Irvine, T.N., and Barager, W.R.A., 1971, A guide to the chemical classification of common volcanic rocks: Canadian Journal of Earth Science, v. 8, p. 523-548.

Kline, J.T., and Bundtzen, T.K., 1986. Two glacial records from westcentral Alaska, in Hamilton, T.D., Reed, K.M., and Thorson, R.M., eds., Glaciation in AlaskaThe geologic record: Anchorage, Alaska Geological Society, p. 123-150.

Krause, K.J., 1984, Photointerpretive maps of morphological floodplain deposits and material resources, middle Kuskokwim River from Sleetmute to Kalskag, Alaska: 
Alaska Division of Geological and Geophysical Surveys Report of Investigations 84-2,4 p., scale 1:63,360, 5 sheets.

Kretschmar, U., and Scout, S.D., 1976, Phase relationships involving arsenopyrite in the system Fe-As-S: Canadian Mineralogist, v. 14, p. 364-386.

Lindgren, Waldemar, 1936, Mineral deposits: New York and London, McGraw-Hill Book Co. Inc., p. 529697.

Maddren, A.G. 1915, Gold placers of the lower Kuskokwim River with a note on copper in the Russian Mountains, in Brooks, A.H., and others, eds. Mineral resources of Alaska-1914: U.S. Geological Survey Bulletin 622. p. $292-360$.

McBirney, A.R., 1984, Igneous petrology: San Francisco, Freeman, Cooger, and Company, $504 \mathrm{p}$.

McGimsey, R.G, and Miller, M.L., 1988, Lahar deposits in the Iditarod volcanics, southwestern Iditarod Quadrangle, in Galloway, J.P., and Hamilton, T.D., ed., Geologic studies in Alaska by the U.S. Geological Survey: U.S. Geological Survey Circular 1016. p. 95-99.

Miller, M.L., Belkin, H.E., Blodgett, R.B., Bundizen, T.K., Cady, J.W., Goldfarb, R.J., Gray, J.E., McGimsey, R.G., and Simpson, S.L., 1989, Pre-field study and mineral resource assessment of the Sleetmute Quadrangle, southwestern Alaska: U.S. Geological Survey Open-file Report 89.363, I15 p., scale 1:250,000. 5 sheets.

Miller, M.L., and Bundtzen, T.K., 1988, Right lateral offset solution for the Iditarod-Nixon Fork fault, in Galloway. J.P., and Hamilton, T.D., eds.. Geologic studies in Alaska by the U.S. Geological Survey: U.S. Geological Survey Circular 1016, p. 99-103.

Orth, D.J., 1971, Dictionary of Alaska place names (2d ed., revised): U.S. Geological Survey Professional Paper 567,1084 p.

Patterson, J.A., 1959, Estimating ore reserves follows logical steps: Engineering and Mining Yournal, v. 160.111, p. 112-115.
Peacock, M.A., 1931, Classification of igneous rock series: Joumal of Gcology. v. 39, p. 54-67.

Reifenstuhl, R.R., Robinson, M.S., Smith, T E., Albanese, M.D., and Allegro, G.A., 1984: Geologic map of the Sleetmute B-6 Quadrangle, Alaska: Alaska Division of Geological and Geophysical Surveys Report of Investigations 84-12, scale 1:40,000, 1 sheet.

Robínson, M.S., Decker, J.E., Reifenstuhl, R.R., Murphy, J.M., and Box, S.E., 1984, Geologic map of the Sleetmute B-5 Quadrangle, Alaska: Alaska Division of Geological and Geophysical Surveys Report of Investigations 84-10, scale 1:40,000, 1 sheet.

Sillitoe, R.H., Halls, Christopher, and Granh J.M., 1975, Porphyry tin deposits in Bolivia: Economic Geology, v. 70, p. 913-927.

Streckeisen, A.B.. 1973, Plutonic rocks, classification and nomenclature recommended by IUGS subcommission on igneous rocks: Gcotines, v. 18, no. 10, p. $26-30$.

Streckeisen, A.B. and LeMaitre, R.W., 1979, A chemical approximation to the modal QAPF classification of the igneous rocks: Stuttgart, N.Jb. Miner. Abh., v. 136, 110. 2, p. 170-206.

Turner, F.J., 1968. Metamorghic petrology: New York, McGraw-Hill Co., 403 p.

Wahrhaftig, Clyde, 1965, Physiographic divisions of Alaska: U.S. Geological Survey Professional Paper 482, $52 \mathrm{p}$.

Waythomas, C.F., 1984, Quaternary glacial sequence in the Chuilnuk and Kiukluk Mountains, Alaska: Geological Society of America Abstracts with Programs, v. 16, no. 5, p. 339.

Wedow, Helmuth, 1953, Preliminary summary of reconnaissance for uranium and thorium in Alaska1952: U.S. Geological Survey Circular 248, 15 p.

West, W.S., 1954, Reconnaissance for radioactive deposits in the lower Yukon-Kuskokwim region, Alaska: U.S. Geological Survey Circular 328, $10 \mathrm{p}$. 